\title{
Estimates and structure of $\alpha$-harmonic functions
}

\author{
Krzysztof Bogdan, Tadeusz Kulczycki† Mateusz Kwaśnicki ${ }^{\ddagger}$
}

$3 / 19 / 2007$

\begin{abstract}
We prove a uniform boundary Harnack inequality for nonnegative harmonic functions of the fractional Laplacian on arbitrary open set $D$. This yields a unique representation of such functions as integrals against measures on $D^{c} \cup\{\infty\}$ satisfying an integrability condition. The corresponding Martin boundary of $D$ is a subset of the Euclidean boundary determined by an integral test.
\end{abstract}

\section{Main results and introduction}

Let $d=1,2, \ldots$, and $0<\alpha<2$. The boundary Harnack principle (BHP) for nonnegative harmonic functions of the fractional Laplacian on $\mathbf{R}^{d}$

$$
\Delta^{\alpha / 2} \varphi(x)=\lim _{\varepsilon \rightarrow 0^{+}} \int_{|y-x|>\varepsilon}[\varphi(y)-\varphi(x)] \nu(x, y) d y,
$$

was proved for Lipschitz domains in 1997 in [9] (compare Theorem 4 below). Here

$$
\nu(x, y)=\mathcal{A}_{d,-\alpha}|y-x|^{-d-\alpha},
$$

$\mathcal{A}_{d, \gamma}=\Gamma((d-\gamma) / 2) /\left(2^{\gamma} \pi^{d / 2}|\Gamma(\gamma / 2)|\right)$ for $-2<\gamma<2$, and, say, $\varphi \in C_{c}^{\infty}\left(\mathbf{R}^{d}\right)$. BHP was extended to all open sets in 1999 in [41], with the constant in the estimate depending on local geometry of their boundary. The question whether the constant may be chosen independently of the domain, or uniformly, was since open.

In what follows $D$ is an arbitrary nonempty open subset of $\mathbf{R}^{d}$ (a domain). Let $G_{D}$ be the Green function of $D$ for $\Delta^{\alpha / 2}$ ([34], [7], [38]). We define the Poisson kernel of $D$ :

$$
P_{D}(x, y)=\int_{D} G_{D}(x, v) \nu(v, y) d v, \quad x \in \mathbf{R}^{d}, y \in D^{c} .
$$

\footnotetext{
*Supported by KBN grant 1 P03A 02629 and RTN contract HPRN-CT-2001-00273-HARP

†Supported by KBN grant 1 P03A 02028 and RTN contract HPRN-CT-2001-00273-HARP

${ }_{\ddagger}^{\ddagger}$ Supported by KBN grant 1 P03A 02028 and RTN contract HPRN-CT-2001-00273-HARP

${ }^{0} 2000$ MS Classification: 31C35, 60J50 (Primary), 31B05, 60G51 (Secondary).

Key words and phrases: boundary Harnack inequality, Martin representation, stable process.
} 
By a calculation of M. Riesz (see [8], [39]), for the ball $B_{r}=\left\{x \in \mathbf{R}^{d}:|x|<r\right\}$ we have

$$
P_{B_{r}}(x, y)=\mathcal{C}_{d, \alpha}\left(\frac{r^{2}-|x|^{2}}{|y|^{2}-r^{2}}\right)^{\alpha / 2} \frac{1}{|x-y|^{d}}, \quad x \in B_{r}, y \in B_{r}^{c},
$$

where $\mathcal{C}_{d, \alpha}=\Gamma(d / 2) \pi^{-1-d / 2} \sin (\pi \alpha / 2)$. Note that if $x$ and $y$ are not too close then $P_{B_{r}}(x, y) \approx\left(r^{2}-|x|^{2}\right)^{\alpha / 2} \cdot\left(|y|^{2}-r^{2}\right)^{-\alpha / 2}|y|^{-d}$ at $\partial B_{r}$. Similar approximate factorization of general $P_{D}$ underlies the following result which is equivalent to the uniform BHP (UBHP) for $\Delta^{\alpha / 2}$ (see also Theorem 4 and Remark 11 below).

Theorem 1 (UBHP) There is a constant $C_{d, \alpha}$, depending only on $d$ and $\alpha$, such that

$$
P_{D}\left(x_{1}, y_{1}\right) P_{D}\left(x_{2}, y_{2}\right) \leq C_{d, \alpha} P_{D}\left(x_{1}, y_{2}\right) P_{D}\left(x_{2}, y_{1}\right)
$$

whenever $r>0, x_{1}, x_{2} \in D \cap B_{r / 2}$ and $y_{1}, y_{2} \in D^{c} \cap B_{r}^{c}$.

Consider the following auxiliary function

$$
s_{D}(x)=\int_{\mathbf{R}^{d}} G_{D}(x, v) d v .
$$

We will say that $y \in \mathbf{R}^{d}$ is accessible from $D$ when

$$
\int_{\mathbf{R}^{d}} s_{D \cap B(y, 1)}(v) \nu(v, y) d v=\infty
$$

or inaccessible when

$$
\int_{\mathbf{R}^{d}} s_{D \cap B(y, 1)}(v) \nu(v, y) d v<\infty .
$$

The point at infinity is called accessible for $D$ if $s_{D}(x)=\infty$ for all $x \in D$, and it is called inaccessible otherwise. Accessibility of a given point from $D$ means that $D$ is rather large near the point, see (17), (75) and the discussion at the end of the paper.

We consider the set $\partial_{*} D$ of limit points of $D$ : we let $\partial_{*} D=\partial D$ if $D$ is bounded and $\partial_{*} D=\partial D \cup\{\infty\}$ if $D$ is unbounded. For unbounded $D, D \ni v \rightarrow \infty$ means that $v \in D$ and $|v| \rightarrow \infty$. We also let $D^{*}=D \cup \partial_{*} D$.

Theorem 1 and Theorem 4 yield estimates of $G_{D}, P_{D}$ and $s_{D}$ at $\partial_{*} D$. Furthermore, the uniformity of the constant yields results on the limits of ratios of these kernel functions. Our main result in this direction is Lemma 8 below. An important consequence of the result is the existence of the Martin kernel. For Greenian $D \subset \mathbf{R}^{d}$ we fix an arbitrary reference point $x_{0} \in D$ and we define the Martin kernel of $D$ :

$$
M_{D}(x, y)=\lim _{D \ni v \rightarrow y} \frac{G_{D}(x, v)}{G_{D}\left(x_{0}, v\right)}, \quad x \in \mathbf{R}^{d}, y \in \partial_{*} D .
$$

Theorem 2 The limit in (8) exists. $M_{D}(x, y)$ is $\alpha$-harmonic in $x$ on $D$ with zero outer charge on $D^{c}$ if and only if $y$ is accessible from $D$. If $y \in \partial D$ is inaccessible then $M_{D}(x, y)=P_{D}(x, y) / P\left(x_{0}, y\right)$. If $\infty \in \partial_{*} D$ is inaccessible then $M_{D}(x, \infty)=$ $s_{D}(x) / s_{D}\left(x_{0}\right)$. 
We define $\partial_{M} D=\left\{y \in \partial_{*} D: y\right.$ is accessible from $\left.D\right\}$ and $D_{M}^{c}=\left\{y \in D^{c}\right.$ : $y$ is inaccessible from $D$ \}. The kernels $M_{D}(\cdot, y), y \in \partial_{M} D$, and $P_{D}(\cdot, y), y \in D_{M}^{c}$, may be used to describe the structure of nonnegative functions harmonic for $\Delta^{\alpha / 2}$ on $D$, or $\alpha$-harmonic (a detailed discussion of the notion of $\alpha$-harmonicity is given in Section 5 ).

Theorem 3 Let $D$ be Greenian. For every function $f \geq 0$ on $D$ which is $\alpha$-harmonic in $D$ with outer charge $\lambda \geq 0$ on $D^{c}$ there is a unique measure $\mu \geq 0$ on $\partial_{M} D$, such that

$$
f(x)=\int_{D^{c}} P_{D}(x, y) \lambda(d y)+\int_{\partial_{M} D} M_{D}(x, y) \mu(d y), \quad x \in D .
$$

As a part of the statement we have that $|\mu|<\infty$,

$$
\int_{D^{c}} P_{D}\left(x_{0}, y\right) \lambda(d y)<\infty
$$

and $\lambda\left(\partial_{M} D\right)=0$, so that $\lambda$ is concentrated on $D_{M}^{c}$, see (76). We remark that for nonGreenian $D$ every such $f$ is constant on $D$, see Lemma 15. For a stronger statement of the uniqueness, see Remark 10 below.

The first integral in (9) reflects the fact that $\Delta^{\alpha / 2}$ is a nonlocal integro-differential operator, allowing for a direct integral-type influence between distant points $x$ and $y$ in the domain of a function, see (1). In particular the role of the boundary condition in the Dirichlet problem of the classical potential theory is now played by a measure ("outer charge") supported on the complement of the domain. Here the generic example is the Poisson kernel $P_{D}(x, y)$ equipped with the Dirac measure at $y \in D^{c}$. We remark that the restriction to harmonicity of only genuine functions would seriously handicap the theory because the limit of a locally bounded pointwise convergent sequence of genuine functions which are harmonic for $\Delta^{\alpha / 2}$ on a given domain may fail to be a genuine $\alpha$-harmonic function itself (see the concluding remark in [36], related to inaccessible boundary points of $D)$.

Theorem 2 and Theorem 3 contrast sharply with the corresponding results in the classical potential theory ([3], [37]), because they are more explicit, and also because the classical Martin kernel is always harmonic, which is no longer the case here. We refer the interested reader to [33] and [18] for a general account on Martin compactification and representation. We also refer to the paper [27], which identifies the classical Martin boundary of Lipschitz domains with their Euclidean boundary (see [37] or [5] for further references). We see that the fractional Laplacian enjoys a similar description in an arbitrary domain.

The role of BHP in explicit determination of the Martin boundary in the classical potential theory is well recognized, see recent [1] and [2] (see also [6], [5], and [3] for more references). The present straightforward derivation of the Martin representation is modeled after [10]. The role of BHP in estimating the Green function and studying Schrödinger-type operators is also well understood. For more information on typical applications we refer the reader to [11], [30], [12], [20], [13], [14], [22], see also [25] for a general perspective.

Our theorems complete and extend in several directions part of the results of [9], [32], [10], [21], [41], [36]. In particular, Theorem 3 was first proved for Lipschitz domains in 
[10] and [21], and for $\kappa$-fat domains in [41]. For these domains all the boundary points are accessible, which influenced the methods of these papers. The first example of what we coin inaccessible boundary point was given in [36]. Our main technical results, Lemma 7 and Lemma 8, develop the ideas of [9] (see also the references in [9]) and [41].

The paper is primarily addressed to the readers interested in the potential theory of nonlocal operators. The theory presently undergoes a rapid development, see [29] and the references given there. The outline and notions which we propose below may likely apply to kernel functions of such operators and the corresponding nonnegative harmonic functions quite generally, except for our treatment of the point at infinity, which is based on Kelvin transformation and therefore is very specific to the present context. Technically, the development hinges on Lemma 7 and Lemma 8 below, and extensions of these should be sought for in the more general settings. A certain role is also played by (22).

Our development is based on M. Riesz' formulas (3) and (23) for the Poisson kernel and the Green function of the ball, and general properties of the Green function and harmonic measure of arbitrary domains, most notably (15). Here our references are [38], [34], and [7]. The reader familiar with the potential theory of Markov processes will notice the relationship of (15) to the strong Markov property of the isotropic $\alpha$-stable Lévy process $\left\{X_{t}, t \geq 0\right\}$ in $\mathbf{R}^{d}$ with the Lévy measure $\nu(0, x) d x$, see [17], [40]. Indeed, probabilistic interpretations and references are our primary source of motivation, as seen from the discussion of such interpretations given at the end of the paper. In the main body of the paper we strive, however, to give elementary and purely analytic definitions and proofs, with a notable exception made for the probabilistic proof of Lemma 10.

The remainder of the paper is organized as follows. In Section 2 we give preliminary definitions and results. In Section 3 we prove Theorem 1 and we state UBHP in a more traditional form as Theorem 4. In Section 4 we study limits of ratios of kernel functions. In Section 5 we define $\alpha$-harmonicity. In Section 6 we prove joint continuity of $M_{D}(x, y)$ and verify Theorem 2. In Section 7 we obtain the Martin representation (9) along with its converse. In Section 8 we prove absolute continuity of harmonic measure on $D_{M}^{c}$, discuss probabilistic interpretations of our results and give examples of accessible and inaccessible boundary points. For instance 0 is inaccessible for $D=\left\{(x, y) \in \mathbf{R}^{2}: y>|x|^{\gamma}\right\}$ if and only if $\gamma<1$.

\section{Preliminaries}

For $x \in \mathbf{R}^{d}$ and $r>0$ we let $|x|=\sqrt{\sum_{i=1}^{d} x_{i}^{2}}, B(x, r)=\{y \in \mathbf{R}:|y-x|<r\}$, $B_{r}=B(0, r)$, and $B=B_{1}$. All the sets, functions and measures considered in the sequel will be Borel. For $U \subset \mathbf{R}^{d}$ we write $U^{c}=\mathbf{R}^{d} \backslash U$. If $k>0$ then $k U=\{k x: x \in U\}$. For a measure $\lambda$ on $\mathbf{R}^{d},|\lambda|$ denotes its total mass. For a function $f$ we let $\lambda(f)=\int f d \lambda$ if the integral makes sense. The probability measure concentrated at $x$ will be denoted by $\varepsilon_{x}$. For nonnegative $f$ and $g$ and a positive number $C$ we write $f \asymp C g$ if $C^{-1} f \leq g \leq C f$. The notation $C_{a, b, \ldots, z}$ means that such constant depends only on $a, b, \ldots, z$. In what follows $U$ will be an arbitrary domain. We will say that $U$ is Greenian if $G_{U}(x, v)$ is finite almost everywhere on $U \times U$. $U$ is always Greenian when $\alpha<d$. If $\alpha \geq d=1$, then $U$ is Greenian if and only if $U^{c}$ is non-polar. In particular, if $\alpha>d=1$, then $U$ is Greenian 
unless $U=\mathbf{R}$. The Green function and the harmonic measure of the fractional Laplacian are defined in [34, Theorem IV.4.16, pp. 229, 240], see also [8], [7, pp. 191, 250, 384], [31], and [38], [17] for the case of dimension one. We will briefly indicate the following crucial properties. If $U$ is Greenian then

$$
\int_{\mathbf{R}^{d}} G_{U}(x, v) \Delta^{\alpha / 2} \varphi(v) d v=-\varphi(x), \quad x \in \mathbf{R}^{d}, \varphi \in C_{c}^{\infty}(U)
$$

Furthermore, $G_{U}(x, v)=G_{U}(v, x)$ for $x, v \in \mathbf{R}^{d}$ ([34, p. 285]). For example, if $\alpha<d$ then (11) is satisfied for $U=\mathbf{R}^{d}$ by the Riesz kernel:

$$
G_{\mathbf{R}^{d}}(x, y)=\mathcal{A}_{d, \alpha}|y-x|^{\alpha-d}, \quad x, y \in \mathbf{R}^{d},
$$

see $\left[34,\left(1.1 .12^{\prime}\right)\right]$, and the harmonic measure, $\omega_{U}^{x}$, is defined as the unique ([34, p. 245], [17]) subprobability measure (probability measure if $D$ is bounded) concentrated on $D^{c}$ such that $\int_{\mathbf{R}^{d}} G_{\mathbf{R}^{d}}(z, y) \omega_{U}^{x}(d z) \leq G_{\mathbf{R}^{d}}(x, y)$ for all $y \in \mathbf{R}^{d}$, and

$$
G_{\mathbf{R}^{d}}(x, y)=\int_{\mathbf{R}^{d}} G_{\mathbf{R}^{d}}(z, y) \omega_{U}^{x}(d z)
$$

for $y \in U^{c}$ except at irregular points of $\partial U$. Recall that a point $y$ is called irregular for $U$ (or thin for $U^{c}$ ) if $\omega_{U}^{y} \neq \varepsilon_{y}$, and it is called regular otherwise, see [7, pp. 348, 272, 353]. Note that "regularity" means here "regularity for the Dirichlet problem on $U$ " [7, p. 348]. The probabilistic interpretation of regularity is that the first hitting time of $U^{c}$ for the corresponding stochastic process starting at $x$ equals zero almost surely, see $[7, \mathrm{p}$. 277]. Note that $y$ is regular for $U$ if and only if $G_{U}(x, y)=0$ for $x \in U$ ([7, Proposition VII.3.1], see also [34, pp. 251, 286]). If $\alpha<d$ then the Green function is given by

$$
G_{U}(x, y)=G_{\mathbf{R}^{d}}(x, y)-\int_{U^{c}} G_{\mathbf{R}^{d}}(z, y) \omega_{U}^{x}(d z) .
$$

For a full discussion we refer the reader to [34], [7] (see also [38] for $1=d \leq \alpha$ ). The harmonic measure may be used to negotiate between Green functions of two domains:

$$
G_{D}(x, v)=G_{U}(x, v)+\int_{\mathbf{R}^{d}} G_{D}(w, v) \omega_{U}^{x}(d w), \quad x, v \in \mathbf{R}^{d}, \text { if } U \subset D,
$$

compare (14). By integrating (15) against the Lebesgue measure we obtain

$$
s_{D}(x)=s_{U}(x)+\int_{\mathbf{R}^{d}} s_{D}(y) \omega_{U}^{x}(d y), \quad x \in \mathbf{R}^{d}, U \subset D .
$$

Clearly,

$$
s_{U} \leq s_{D}, \quad \text { if } U \subset D .
$$

Recall that $\operatorname{supp} \omega_{U}^{x} \subset U^{c}, x \in \mathbf{R}^{d}$. If $U \subset D$ then

$$
\omega_{D}^{x}(A)=\omega_{U}^{x}(A)+\int_{D \backslash U} \omega_{D}^{y}(A) \omega_{U}^{x}(d y), \quad A \subset D^{c},
$$


in particular $G_{U}(x, v) \leq G_{D}(x, v)$ and for $A \subset D^{c}, x, v \in U$, we have

$$
\omega_{U}^{x}(A) \leq \omega_{D}^{x}(A) .
$$

Furthermore, if $D_{1} \subset D_{2} \subset \ldots$ and $D=\bigcup D_{n}$, then $G_{D_{n}}(x, v) \uparrow G_{D}(x, v)$ and $\omega_{D_{n}}^{x}(\varphi) \rightarrow$ $\omega_{D}^{x}(\varphi)$ whenever $x, v \in D$ and $\varphi \in C_{0}\left(\mathbf{R}^{d}\right)$ (vague convergence, weak convergence for bounded $D[34,(4.6 .6)])$.

Let $\varphi \in C_{c}^{\infty}\left(\mathbf{R}^{d}\right)$ and let open Greenian $D^{\prime}$ contain both $D$ and the support of $\varphi$. Using (11) for $D$ and $D^{\prime},(15)$, and Fubini we obtain

$$
\int_{D} G_{D}(x, v) \Delta^{\alpha / 2} \varphi(v) d v=\int_{D^{c}}[\varphi(y)-\varphi(x)] \omega_{D}^{x}(d y), \quad x \in D, \varphi \in C_{c}^{\infty}\left(\mathbf{R}^{d}\right) .
$$

By considering $\varphi$ supported away from $\bar{D}$, and by (1) we conclude that on $(\bar{D})^{c}, \omega_{D}^{x}$ is absolutely continuous with respect to the Lebesgue measure, and has density $P_{D}(x, y)$ given by (2). This is the Ikeda-Watanabe formula ([28]):

$$
\omega_{D}^{x}(A)=\int_{A} P_{D}(x, y) d y, \quad \text { if } \operatorname{dist}(A, D)>0 .
$$

If $D^{\prime} \supset D$ is a Lipschitz domain (e.g. a ball) then $\omega_{D}^{x}\left(\partial D^{\prime}\right) \leq \omega_{D^{\prime}}^{x}\left(\partial D^{\prime}\right)=0([9])$, hence

$$
\omega_{D}^{x}(d y)=P_{D}(x, y) d y \text { on } D^{\prime c} \text { provided } x \in D \subset D^{\prime} \text { and } D^{\prime} \text { is Lipschitz } .
$$

The Green function of the ball is known explicitly:

$$
G_{B_{r}}(x, v)=\mathcal{B}_{d, \alpha}|x-v|^{\alpha-d} \int_{0}^{w} \frac{s^{\alpha / 2-1}}{(s+1)^{d / 2}} d s, \quad x, v \in B_{r}
$$

where

$$
w=\left(r^{2}-|x|^{2}\right)\left(r^{2}-|v|^{2}\right) /|x-v|^{2},
$$

and $\mathcal{B}_{d, \alpha}=\Gamma(d / 2) /\left(2^{\alpha} \pi^{d / 2}[\Gamma(\alpha / 2)]^{2}\right)$, see [8], [39]. It is also known ([14], [19]) that

$$
s_{B_{r}}(x)=\frac{\mathcal{C}_{d, \alpha}}{\mathcal{A}_{d,-\alpha}}\left(r^{2}-|x|^{2}\right)^{\alpha / 2}, \quad|x| \leq r .
$$

For a nonnegative measure $\lambda$ on $\mathbf{R}^{d}\left(D^{c}\right)$ we define its Poisson integral on $D$,

$$
P_{D}[\lambda](x)=\int_{D^{c}} P_{D}(x, y) \lambda(d y), \quad x \in D,
$$

compare (9). To simultaneously control $P_{D}[\lambda]$ and $\lambda$ we define the measure

$$
P_{D}^{*}[\lambda](d x)=P_{D}[\lambda](x) d x+\lambda(d x) .
$$

Thus, $P_{D}^{*}[\lambda]$ is equal to $\lambda$ on $D^{c}$, and on $D$ it is absolutely continuous with respect to the Lebesgue measure, with $P_{D}[\lambda]$ as the density function. Of course, $P_{D}\left[P_{D}^{*}[\lambda]\right](x)=$ $P_{D}[\lambda](x), x \in D$. This observation will be strengthened in (27) below. 
If $U \subset D$ and $v \in U^{c}$ is such that $G_{U}(x, v)=0$ for $x \in \mathbf{R}^{d}$ (in particular, if $U$ is a Lipschitz domain and $v \in U^{c}$ is arbitrary), then by (15) we have

$$
G_{D}(x, v)=\int G_{D}(w, v) \omega_{U}^{x}(d w), \quad x \in U
$$

This, and (28) below may be considered a mean value property.

The following sum of integrals will be important. Consider a nonnegative function $f$ on $D$, a nonnegative measure $\lambda$ on $D^{c}$ and a nonempty open set $U \subset D$. We denote

$$
\Omega_{U}^{D}[f, \lambda](x)=\int_{D \backslash U} f(y) \omega_{U}^{x}(d y)+\int_{D^{c}} P_{U}(x, y) \lambda(d y), \quad x \in U .
$$

Informally, we may think of $\Omega_{U}^{D}[f, \lambda]$ as an integral of $f+\lambda$ against the harmonic measure $\omega_{U}$. The delicate point of the definition is that the integration over $\partial U \cap \partial D$ is restricted to the part of the harmonic measure which is absolutely continuous with respect to the Lebesgue measure, with the density function given by the Poisson kernel. The convention will play a role for $U$ touching $\partial D$. In this connection see (21), Proposition 1 and the discussion at the end of the paper.

Lemma 1 If $U \subset D$ and $\lambda$ is a nonnegative measure on $D^{c}$, then

$$
P_{D}[\lambda](x)=\Omega_{U}^{D}\left[P_{D}[\lambda], \lambda\right](x), \quad x \in U .
$$

Proof: Let $x \in U, y \in D^{c}$. By integrating (15) against $\nu(v, y) d v$ on $\mathbf{R}^{d}$, and (2), we get

$$
P_{D}\left[\varepsilon_{y}\right](x)=P_{D}(x, y)=P_{U}(x, y)+\int P_{D}(z, y) \omega_{U}^{x}(d z)=\Omega_{U}^{D}\left[P_{D}\left[\varepsilon_{y}\right], \varepsilon_{y}\right](x) .
$$

The case of general $\lambda \geq 0$ follows from Fubini-Tonelli theorem.

The next two lemmas are versions of Harnack inequality, see also Remark 8.

Lemma 2 If $\lambda \geq 0$ and $x_{1}, x_{2} \in B_{r} \subset B_{s} \subset D$ then

$$
P_{D}[\lambda]\left(x_{1}\right) \leq\left(\frac{1+r / s}{1-r / s}\right)^{d} P_{D}[\lambda]\left(x_{2}\right) .
$$

Proof: By (3) we have $P_{B_{s}}\left(x_{1}, z\right) \leq(1+r / s)^{d}(1-r / s)^{-d} P_{B_{s}}\left(x_{2}, z\right)$ if $|z| \geq s$. Using the second equality in (29) with $U=B_{s},(22)$, and (3), we prove the result.

Lemma 3 If $x_{1}, x_{2} \in D$ then there is $c_{x_{1}, x_{2}}$ such that for every $\lambda \geq 0$

$$
P_{D}[\lambda]\left(x_{1}\right) \leq c_{x_{1}, x_{2}} P_{D}[\lambda]\left(x_{2}\right) .
$$

Proof: If $x_{1}, x_{2} \in B_{r} \subset B_{2 r} \subset D$ for some $r>0$ then we are done by Lemma 2 with $c=c_{x_{1}, x_{2}}$ depending only on $d$. Assume that $B\left(x_{1}, 2 r\right) \subset D, B\left(x_{2}, 2 r\right) \subset D, B\left(x_{1}, 2 r\right) \cap$ $B\left(x_{2}, 2 r\right)=\emptyset$ for some $r>0$, and consider (29) with $U=B\left(x_{1}, r\right)$. Let $y \in D^{c}$. By $(22)$ and the first part of the proof we obtain $P_{D}\left(x_{1}, y\right) \geq \int_{B\left(x_{2}, r\right)} c P_{D}\left(x_{2}, y\right) P_{B_{r}}\left(0, x-x_{1}\right) d x$.

If $K \subset D$ is compact and $x_{1}, x_{2} \in K$ then $c_{x_{1}, x_{2}}$ in Harnack's inequality above may be so chosen to depend only on $K, D$, and $\alpha$, because $r$ in the above proof may be chosen independently of $x_{1}, x_{2}$. Note that $D$ and $K$ may be disconnected. 
Remark 1 If $\lambda \geq 0$ and $P_{D}[\lambda](x)$ is finite (positive) for some $x \in D$, then it is locally bounded from above (below, resp.) for all $x \in D$. This follows from Lemma 3. Note that if (10) holds then $P_{D}[\lambda]$ is finite and locally uniformly Lipschitz continuous on $D$, a consequence of (30).

The following well-known result is given for the reader's convenience.

Lemma $4 G_{D}$ is positive and jointly continuous: $D \times D \mapsto(0, \infty]$.

Proof: By (26), (22), Lemma 2 and symmetry, $G_{D}$ is locally bounded on $\{(x, y) \in$ $D \times D: x \neq y\}$. By Remark $1, G_{D}$ is locally uniformly continuous in each variable, and so it is jointly continuous on this set. Near the diagonal $\{(x, x): x \in D\}$ we use (15) with $U=B(x, s) \subset D$. For this $U$ the first term on the right hand side of (15) is explicitly given by (23) and also positive on $U \times U$ and the second term can be dealt with as before. Thus, by Lemma $3, G_{D}(x, y)$ is jointly continuous $D \times D \mapsto[0, \infty]$ and $G_{D}(x, y)>0$ on $D \times D$, regardless of connectedness of $D$.

For clarity we note that $G_{D}$ is finite and locally uniformly continuous on $D \times D \backslash\{(x, x)$ : $x \in D\}$ (on $D \times D$ if $\alpha>d=1$ ), see (15) and (23).

Scaling will be important in what follows. Let $k>0$. We have

$$
\int_{k U} \nu(0, y) d y=k^{-\alpha} \int_{U} \nu(0, y) d y
$$

Similarly, if $\varphi_{k}(x)=\varphi(x / k)$ and $\varphi \in C_{c}^{\infty}\left(\mathbf{R}^{d}\right)$ then

$$
\Delta^{\alpha / 2} \varphi_{k}(x)=k^{-\alpha} \Delta^{\alpha / 2} \varphi(x / k), \quad x \in \mathbf{R}^{d} .
$$

By (11) and uniqueness of the Green function we see that

$$
G_{k U}(k x, k v)=k^{\alpha-d} G_{U}(x, v), \quad x, v \in \mathbf{R}^{d},
$$

hence

$$
s_{k U}(k x)=k^{\alpha} s_{U}(x), \quad x \in \mathbf{R}^{d},
$$

and

$$
P_{k D}(k x, k y)=k^{-d} P_{D}(x, y), \quad x, y \in \mathbf{R}^{d} .
$$

By (20) we also have that

$$
\omega_{k D}^{k x}(k A)=\omega_{D}^{x}(A), \quad x \in \mathbf{R}^{d}, A \subset \mathbf{R}^{d} .
$$

Translation invariance is equally important but easier to observe, for example we have $G_{U+y}(x+y, v+y)=G_{U}(x, v)$. Both properties enable us to reduce many of the considerations below to the setting of the unit ball centered at the origin. 


\section{Approximate factorization of Poisson kernel}

We keep assuming that $\emptyset \neq D \subset \mathbf{R}^{d}$ is open. Note that the constants in the estimates below are independent of $D$. When $0<r \leq 1$ we denote $D_{r}=D \cap B_{r}$ and $D_{r}^{\prime}=B^{c} \cup D \backslash B_{r}$. Our first estimate is an extension of an observation made in [41, the proof of Lemma 3.3].

Lemma 5 For every $p \in(0,1)$ there is a constant $C_{d, \alpha, p}$ such that if $D \subset B$ then

$$
\omega_{D}^{x}\left(B^{c}\right) \leq C_{d, \alpha, p} s_{D}(x), \quad x \in D_{p} .
$$

Proof: Let $0<p<1$. We choose a function $\varphi \in C_{c}^{\infty}\left(\mathbf{R}^{d}\right)$ such that $0 \leq \varphi \leq 1, \varphi(y)=1$ if $|y| \leq p$, and $\varphi(y)=0$ if $|y| \geq 1$. Let $x \in D_{p}$. By (20) we have

$$
\begin{aligned}
\omega_{D}^{x}\left(B^{c}\right) & =\int_{B^{c}}(\varphi(x)-\varphi(y)) \omega_{D}^{x}(d y) \leq \int_{D^{c}}(\varphi(x)-\varphi(y)) \omega_{D}^{x}(d y) \\
& =-\int_{D} G_{D}(x, y) \Delta^{\alpha / 2} \varphi(y) d y .
\end{aligned}
$$

It remains to observe that $\Delta^{\alpha / 2} \varphi$ is bounded and the lemma follows.

For $x \in \mathbf{R}^{d}, r>0$, and a nonnegative measure $\lambda$ on $\mathbf{R}^{d}$, we let

$$
\Lambda_{x}(\lambda)=\int_{\mathbf{R}^{d}} \nu(x, y) \lambda(d y), \quad \text { and } \quad \Lambda_{x, r}(\lambda)=\int_{B(x, r)^{c}} \nu(x, y) \lambda(d y) .
$$

Note that if $k>0$ and $\lambda_{k}$ is the dilation of the measure $\lambda$ defined by

$$
\int \varphi(y) \lambda_{k}(d y)=k^{d} \int \varphi(k y) \lambda(d y)
$$

then

$$
\Lambda_{0, k r}\left(\lambda_{k}\right)=k^{-\alpha} \Lambda_{0, r}(\lambda)
$$

Lemma 6 Let $0<p<1$. There is $C_{d, \alpha, p}$ such that if $D \subset B, \lambda \geq 0, \operatorname{supp} \lambda \subset B^{c}$, then

$$
P_{D}[\lambda](x) \leq C_{d, \alpha, p} \Lambda_{0, p}\left(P_{D}^{*}[\lambda]\right), \quad x \in D_{p} .
$$

Proof: Let $0<p<q<r \leq 1$ and $x \in D_{p}$. By (28) and (19) we have

$$
P_{D}[\lambda](x)=\Omega_{D_{r}}^{D}\left[P_{D}[\lambda], \lambda\right](x) \leq \int_{D_{r}^{\prime}} P_{B_{r}}(x, y) P_{D}^{*}[\lambda](d y) .
$$

Fubini-Tonelli theorem yields

$$
P_{D}[\lambda](x) \leq \frac{1}{1-q} \int_{q}^{1} \int_{D_{r}^{\prime}} P_{B_{r}}(x, y) P_{D}^{*}[\lambda](d y) d r=\int_{D_{q}^{\prime}} K(x, y) P_{D}^{*}[\lambda](d y),
$$

where, according to (3),

$$
K(x, y)=\frac{1}{1-q} \int_{q}^{1 \wedge|y|} P_{B_{r}}(x, y) d r=\frac{\mathcal{C}_{d, \alpha}}{1-q} \int_{q}^{1 \wedge|y|}\left(\frac{r^{2}-|x|^{2}}{|y|^{2}-r^{2}}\right)^{\alpha / 2} \frac{1}{|x-y|^{d}} d r .
$$


Here and below $|y| \geq q$ and $r \leq 1 \wedge|y|$, which implies that

$$
\frac{|x-y|}{|y|} \geq \frac{q-p}{q}, \quad \frac{|y|+r}{|y|} \geq 1, \text { and } \quad r^{2}-|x|^{2} \leq 1
$$

Thus

$$
K(x, y) \leq \frac{C_{d, \alpha, q / p}}{|y|^{d+\alpha / 2}} \int_{q}^{1 \wedge|y|} \frac{d r}{(|y|-r)^{\alpha / 2}} \leq \frac{C_{d, \alpha, q / p}}{|y|^{d+\alpha}}
$$

We conclude the proof by choosing, e.g., $q=(1+p) / 2$.

The above regularization of $P_{B_{r}}(x, y)([9])$ is an analogue of volume averaging in classical potential theory.

Lemma 7 Let $0<p<1$. There is $C_{d, \alpha, p}$ such that if $\lambda \geq 0$, supp $\lambda \subset B^{c}$ and $D \subset B$, then

$$
C_{d, \alpha, p}^{-1} \Lambda_{0, p}\left(P_{D}^{*}[\lambda]\right) s_{D}(x) \leq P_{D}[\lambda](x) \leq C_{d, \alpha, p} \Lambda_{0, p}\left(P_{D}^{*}[\lambda]\right) s_{D}(x), \quad x \in D_{p} .
$$

Proof: Let $0<p<q<r<1$ and $x \in D_{p}$. By (28) and (21) we have that

$$
P_{D}[\lambda](x)=\int_{D_{r}^{\prime}} P_{D_{q}}(x, y) P_{D}^{*}[\lambda](d y)+\int_{D_{r} \backslash D_{q}} P_{D}[\lambda](y) \omega_{D_{q}}^{x}(d y)
$$

If $v \in D_{q}$ and $y \in B_{r}^{c}$, then $(r-q) / r \leq|y-v| /|y| \leq(r+q) / q$. Hence

$$
\begin{aligned}
\int_{D_{r}^{\prime}} P_{D_{q}}(x, y) P_{D}^{*}[\lambda](d y) & =\int_{D_{r}^{\prime}} \int_{D_{q}} G_{D_{q}}(x, v) \nu(v, y) d v P_{D}^{*}[\lambda](d y) \\
& \asymp C_{d, \alpha, r, q} s_{D_{q}}(x) \int_{D_{r}^{\prime}} \nu(0, y) P_{D}^{*}[\lambda](d y) .
\end{aligned}
$$

The second integral of (40) is estimated by using Lemma 5,6 , and scaling $(35,63)$ :

$$
\begin{aligned}
\int_{D_{r} \backslash D_{q}} P_{D}[\lambda](y) \omega_{D_{q}}^{x}(d y) & \leq \omega_{D_{q}}^{x}\left(B_{q}^{c}\right) \sup _{D_{r} \backslash D_{q}} P_{D}[\lambda] \\
& \leq C_{d, \alpha, p, q, r} s_{D_{q}}(x) \int_{D_{r}^{\prime}} \nu(0, y) P_{D}^{*}[\lambda](d y) .
\end{aligned}
$$

Since $P_{D}[\lambda]$ is nonnegative, (40), (41) and (42) yield:

$$
P_{D}[\lambda](x) \asymp C_{d, \alpha, p, q, r} s_{D_{q}}(x) \int_{D_{r}^{\prime}} \nu(0, y) P_{D}^{*}[\lambda](d y) .
$$

Clearly, $s_{D_{q}}(x) \leq s_{D}(x)$. In view of (16), Lemma 5 and scaling we also have that

$$
\begin{aligned}
s_{D}(x) & =s_{D_{q}}(x)+\int_{D \backslash D_{q}} s_{D}(z) \omega_{D_{q}}^{x}(d z) \leq s_{D_{q}}(x)+\omega_{D_{q}}^{x}\left(B_{q}^{c}\right) \sup _{D} s_{D} \\
& \leq s_{D_{q}}(x)\left(1+C_{d, \alpha, p, q} \sup _{B} s_{B}\right)=C_{d, \alpha, p, q} s_{D_{q}}(x) .
\end{aligned}
$$


Of course, $\int_{D_{r}^{\prime}} \nu(0, y) P_{D}^{*}[\lambda](d y) \leq \int_{B_{p}^{c}} \nu(0, y) P_{D}^{*}[\lambda](d y)$. Lemma 6 yields that also

$$
\begin{aligned}
\int_{B_{p}^{c}} \nu(0, y) P_{D}^{*}[\lambda](d y) & \leq \int_{D_{r}^{\prime}} \nu(0, y) P_{D}^{*}[\lambda](d y)+\frac{C_{d, \alpha}\left|D_{r}\right|}{p^{d+\alpha}} \sup _{D_{r}} P_{D}[\lambda] \\
& \leq C_{d, \alpha, p, r} \int_{D_{r}^{\prime}} \nu(0, y) P_{D}^{*}[\lambda](d y) .
\end{aligned}
$$

This proves (39) by choosing, e.g., $q=p+(1-p) / 3$ and $r=p+2(1-p) / 3$. In fact for every $x \in D$ we have

$$
P_{D}[\lambda](x)=\int_{B^{c}} \int_{D} G_{D}(x, z) \nu(z, y) d z P_{D}^{*}[\lambda](d y) \geq C_{d, \alpha} s_{D}(x) \int_{B^{c}} \nu(0, y) P_{D}^{*}[\lambda](d y) .
$$

Remark 2 Scaling leaves (39) invariant. Indeed, let $\lambda_{k}$ be defined by (36) for some $k>0$. By $(35,34), P_{k D}\left[\lambda_{k}\right](k x)=P_{D}[\lambda](x)$ for $x \in D$. By (37) and (63) we have that $\Lambda_{0, k p}\left(P_{k D}^{*}\left[\lambda_{k}\right]\right) s_{k D}(k x)=\Lambda_{0, p}\left(P_{D}^{*}[\lambda]\right) s_{D}(x)$, which is our claim. Similar observation is valid for translation.

Remark 3 The constant $C_{d, \alpha, p}$ in (39) may be considered nondecreasing in $p$. Indeed, if $0<p_{1}<p_{2}<1$ and $P_{D}[\lambda] \leq C_{d, \alpha, p_{2}} \Lambda_{0, p_{2}}\left(P_{D}^{*}[\lambda]\right) s_{D}$ on $D_{p_{2}}$ then $P_{D}[\lambda] \leq$ $C_{d, \alpha, p_{2}} \Lambda_{0, p_{1}}\left(P_{D}^{*}[\lambda]\right) s_{D}$ on $D_{p_{1}}$. Similarly, if $C_{d, \alpha, p_{1}}^{-1} \Lambda_{0, p_{1}}\left(P_{D}^{*}[\lambda]\right) s_{D} \leq P_{D}[\lambda]$ on $D_{p_{1}}$, then $C_{d, \alpha, p_{2}}^{-1} \Lambda_{0, p_{1}}\left(P_{D}^{*}[\lambda]\right) s_{D} \leq P_{D}[\lambda]$ on $D_{p_{1}}$.

The lower bound in (39) even holds with a constant independent of $p$, see (43).

Proof of Theorem 1: By (34) we only need to consider $r=1$. Let $D_{1}=D \cap B$. By Lemma 1 and (22), for $i, j=1,2$ we have that $P_{D}\left(x_{i}, y_{j}\right)=P_{D_{1}}\left[\lambda_{j}\right]\left(x_{i}\right)$, where $\lambda_{j} \geq 0$. Lemma 7 with $p=1 / 2$ yields

$$
\begin{aligned}
P_{D}\left(x_{1}, y_{1}\right) P_{D}\left(x_{2}, y_{2}\right) & \leq C_{d, \alpha, 1 / 2}^{2} \Lambda_{0,1 / 2}\left(P_{D_{1}}^{*}\left[\lambda_{1}\right]\right) s_{D_{1}}\left(x_{1}\right) \Lambda_{0,1 / 2}\left(P_{D_{1}}^{*}\left[\lambda_{2}\right]\right) s_{D_{1}}\left(x_{2}\right) \\
& \leq C_{d, \alpha, 1 / 2}^{4} P_{D}\left(x_{1}, y_{2}\right) P_{D}\left(x_{2}, y_{1}\right) .
\end{aligned}
$$

Let $\lambda, \rho$ be nonnegative measures on $B_{r}^{c}$. Integrating (4) with respect to $\lambda\left(d y_{1}\right) \rho\left(d y_{2}\right)$, for $x_{1}, x_{2} \in D \cap B_{r / 2}$ we obtain

$$
P_{D}[\lambda]\left(x_{1}\right) P_{D}[\rho]\left(x_{2}\right) \leq C_{d, \alpha} P_{D}[\rho]\left(x_{1}\right) P_{D}[\lambda]\left(x_{2}\right) .
$$

By translation, (44) and (4) extend to intersections of $D$ and balls of arbitrary center.

Inequality (44) and the following global version of it state our uniform BHP in a more traditional form, see also Remark 11. We emphasize that the constant in (46) below does not depend on $D$, and that $D$ may be disconnected.

Theorem 4 Let $G \subset \mathbf{R}^{d}$ be open and let $K \subset G$ be compact. There is a constant $C=C_{d, \alpha, G, K}$ with the following property. If $D \subset \mathbf{R}^{d}$ is open, $\lambda, \rho$ are nonnegative measures not charging $G \cap D^{c}$, and $f=P_{D}[\lambda], g=P_{D}[\rho]$, then

$$
C^{-1} f(y) g(x) \leq f(x) g(y) \leq C f(y) g(x), \quad x, y \in K \cap D .
$$


PROOF: In what follows will use Lemma 7 and a refinement of the argument used in the proof of the global Harnack inequality (Lemma 3). For every $x \in K$ we consider a ball $B\left(x, r_{x}\right) \subset G$. We select a finite covering, $B\left(x_{1}, p r_{x_{1}}\right), \ldots, B\left(x_{n}, p r_{x_{n}}\right)$, of $K$, where, e.g., $p=1 / 2$. We denote $r_{j}=r_{x_{j}}, B_{j}=B\left(x_{j}, r_{j}\right), \widetilde{B}_{j}=B\left(x_{j}, p r_{j}\right)$, where $j=1, \ldots, n$, and we let $R=\operatorname{diam} K$ and $r=\min \left\{r_{1}, \ldots, r_{n}\right\}$. We now fix $x, y \in D \cap K$ and let $i, j$ be such that $x \in D \cap \widetilde{B}_{i}, y \in D \cap \widetilde{B}_{j}$. Let $f=P_{D}[\lambda]$, as described above. Note that $f$ is a Poisson integral on each $D \cap B_{i}$ by (28) and (22). By Lemma 7 and Remark 2 we obtain

$$
\frac{f(x)}{s_{D \cap B_{i}}(x)} \leq C_{d, \alpha, p}\left(\int_{\widetilde{B}_{i}^{c} \backslash \widetilde{B}_{j}} \nu\left(x_{i}, z\right) P_{D}^{*}[\lambda](d z)+\int_{\widetilde{B}_{i}^{c} \cap \widetilde{B}_{j} \cap D} \nu\left(x_{i}, z\right) f(z) d z\right) .
$$

For $z \in \widetilde{B}_{i}^{c} \backslash \widetilde{B}_{j}$ we have $\left|z-x_{j}\right| \leq R+\left|z-x_{i}\right| \leq \frac{R+r p}{r p}\left|z-x_{i}\right|$, thus $\nu\left(x_{i}, z\right) \leq$ $C_{d, \alpha, p, r, R} \nu\left(x_{j}, z\right)$ in the first integral. In the second one we simply estimate $\nu\left(x_{i}, z\right) \leq$ $C_{d, \alpha, p, r}$. It follows that

$$
\frac{f(x)}{s_{D \cap B_{i}}(x)} \leq C_{d, \alpha, p, r, R}\left(\int_{\widetilde{B}_{j}^{c}} \nu\left(x_{j}, z\right) P_{D}^{*}[\lambda](d z)+\int_{D \cap \widetilde{B}_{j}} f(z) d z\right) .
$$

We use Lemma 7 and Remark 2 to estimate the integrals. We obtain

$$
\frac{f(x)}{s_{D \cap B_{i}}(x)} \leq C_{d, \alpha, p, r, R}\left(\frac{f(y)}{s_{D \cap B_{j}}(y)}+\Lambda_{x_{j}, p r_{j}}\left(P_{D}^{*}[\lambda]\right) \int_{D \cap \widetilde{B}_{j}} s_{D \cap B_{j}}(z) d z\right) .
$$

But $s_{D \cap B_{j}} \leq s_{B_{j}} \leq C_{d, \alpha, R}$, and $\Lambda_{x_{j}, p r_{j}}\left(P_{D}^{*}[\lambda]\right) \leq C_{d, \alpha, p} f(y) / s_{D \cap B_{j}}(y)$. Therefore

$$
\frac{f(x)}{s_{D \cap B_{i}}(x)} \leq C_{d, \alpha, p, r, R} \frac{f(y)}{s_{D \cap B_{j}}(y)} .
$$

By analogous inequality for $g$ we obtain (45).

Remark 4 We note that (45) may be written as

$$
C^{-1} \frac{f(y)}{g(y)} \leq \frac{f(x)}{g(x)} \leq C \frac{f(y)}{g(y)}, \quad x, y \in D \cap K,
$$

provided the Poison integrals are nonzero and finite for (one and therefore for all) $x \in D$. Specifically, for $f$ the condition means that (10) holds and $\lambda$ is not equal to zero on $D^{c}$.

Remark 5 As seen in the above proof, $C$ in (46) depends only on $d, \alpha$, diam $G$ and $\operatorname{dist}\left(K, G^{c}\right)$. In fact, by scaling, its dependence on $\operatorname{diam} G$ and $\operatorname{dist}\left(K, G^{c}\right)$ is only through the ratio $\operatorname{dist}\left(K, G^{c}\right) / \operatorname{diam} G$.

Remark 6 Let $D \subset \mathbf{R}^{d}$ be open, $U \subset D$ bounded, $f=P_{D}[\lambda]$ for a nonnegative measure $\lambda$ on $D^{c}$. If $h=\operatorname{dist}(\operatorname{supp} \lambda, U)>0$ and $f$ is finite at one point of $U$ then $f$ is bounded on $U$. This follows from Theorem 4 applied to $K=\bar{U}, G=\left\{x \in \mathbf{R}^{d}: \operatorname{dist}(x, U)<h / 3\right\}$ and $g=P_{D \cap G}\left[\mathbf{1}_{A} d x\right] \leq 1$, where $A=\left\{x \in \mathbf{R}^{d}: \operatorname{dist}(x, U)>2 h / 3\right\}$ is open and nonempty. 
Remark 7 Let $D \subset \mathbf{R}^{d}$ be an open Greenian set, let $x_{0} \in D$ and $f(x)=G_{D}\left(x, x_{0}\right)$. It is well known that the set $\{x \in \partial D: f(x)>0\}$ is polar ([34, p.263]) so it is of Lebesgue measure zero. Let $G$ be an open bounded Lipschitz domain, $D \cap G \neq \emptyset$, and assume that $x_{0} \notin G$. Then $\omega_{G}^{x}(\partial G)=0$ for $x \in G$. By the above and (26) we have

$$
f(x)=\int_{D \backslash \bar{G}} f(w) P_{D \cap G}(x, w) d w, \quad x \in D \cap G,
$$

so $f$ is a Poisson integral on $D \cap G$. We thus may apply Theorem 4 to $D, G$ and $f$ as above. We may also use Remark 6 for $f$. It follows that for arbitrary $r>0$ the function is bounded on any bounded subset of $D \backslash B\left(x_{0}, r\right)$. The result is nontrivial if $d \leq \alpha$, especially for $d=\alpha=1$.

\section{Existence of limits}

For a positive function $q$ on a nonempty set $U$ we define its relative oscillation:

$$
\mathrm{RO}_{U} q=\mathrm{RO}_{x \in U} q(x)=\frac{\sup _{x \in U} q(x)}{\inf _{x \in U} q(x)} .
$$

For notational convenience, we put $\mathrm{RO}_{U} q=1$ if $U=\emptyset$.

The main result of this section addresses the asymptotics of Poisson integrals at $x=0$. (30) gives a motivation for (47), but here $x=0$ may be, e.g., a boundary point of $D$.

Lemma 8 For every $\eta>0$ there exists $r>0$ such that

$$
\mathrm{RO}_{D \cap B_{r}} \frac{P_{D}\left[\lambda_{1}\right]}{P_{D}\left[\lambda_{2}\right]} \leq 1+\eta
$$

for all open $D \subset B$ and nonzero nonnegative measures $\lambda_{1}, \lambda_{2}$ on $B^{c}$ satisfying (10).

Proof: Let $c$ denote $C_{d, \alpha, 1 / 2}$ of Lemma 7 with $p=1 / 2$. Recall from the proof of Theorem 1 that (44) holds with $C_{d, \alpha}=c^{4}$. Thus, (47) holds for $r=1 / 2$ with $1+\eta$ replaced by $c^{4}$. We will show that the left hand side of (47) is self-improving when $r \rightarrow 0^{+}$. This will be done under each of the two complementary assumptions: (49) and (53) below. First, however, we need some preparation. For $0<p<q<1 / 2$ and a measure $\lambda$ let $D_{p, q}=D_{q} \backslash D_{p}$ and

$$
\Lambda_{x, p, q}(\lambda)=\int_{D_{p, q}} \nu(x, y) \lambda(d y) .
$$

We also denote

$$
\begin{array}{lll}
f_{i}=P_{D}\left[\lambda_{i}\right], & f_{i}^{p, q}=P_{D_{p}}\left[\mathbf{1}_{D_{p, q}} P_{D}^{*}\left[\lambda_{i}\right]\right], & \widetilde{f}_{i}^{p, q}=P_{D_{p}}\left[\mathbf{1}_{D_{q}^{\prime}} P_{D}^{*}\left[\lambda_{i}\right]\right], \\
f_{i}^{*}=P_{D}^{*}\left[\lambda_{i}\right], & f_{i}^{p, q *}=P_{D_{p}}^{*}\left[\mathbf{1}_{D_{p, q}} P_{D}^{*}\left[\lambda_{i}\right]\right], & \widetilde{f}_{i}^{p, q *}=P_{D_{p}}^{*}\left[\mathbf{1}_{D_{q}^{\prime}} P_{D}^{*}\left[\lambda_{i}\right]\right] .
\end{array}
$$

What follows will be valid for $i=1$ and $i=2$. By (28) and (22) we have $f_{i}=f_{i}^{p, q}+\widetilde{f}_{i}^{p, q}$ and $f_{i}^{*}=f_{i}^{p, q *}+\widetilde{f}_{i}^{p, q *}$. For $r \in(0,1 / 2]$ we denote $m_{r}=\inf _{D_{r}}\left(f_{1} / f_{2}\right)$ and $M_{r}=\sup _{D_{r}}\left(f_{1} / f_{2}\right)$. As we noted above, $M_{r} \leq c^{4} m_{r}$. Let $\varepsilon>0$. 
Let $q \in(0,1 / 2]$ and let $p=p(q) \in(0, q / 2)$ (depending on $q$ and $\varepsilon$ ) be defined by

$$
(q+2 p) /(q-2 p)=1+\varepsilon,
$$

so that if $z \in D_{2 p}$ and $y \in B_{q}^{c}$ then $(1+\varepsilon)^{-d-\alpha} \nu(0, y) \leq \nu(z, y) \leq(1+\varepsilon)^{d+\alpha} \nu(0, y)$. Thus, for $x \in D_{2 p}$ we have

$$
\widetilde{f}_{i}^{2 p, q}(x)=\int_{D_{q}^{\prime}} \int_{D_{2 p}} G_{D_{2 p}}(x, z) \nu(z, y) d z f_{i}^{*}(d y) \leq(1+\varepsilon)^{d+\alpha} \Lambda_{0, q}\left(f_{i}^{*}\right) s_{D_{2 p}}(x),
$$

and

$$
\widetilde{f}_{i}^{2 p, q}(x) \geq(1+\varepsilon)^{-d-\alpha} \Lambda_{0, q}\left(f_{i}^{*}\right) s_{D_{2 p}}(x) .
$$

We will now examine consequences of the following assumption:

$$
\Lambda_{0, p, q}\left(f_{i}^{*}\right) \leq \varepsilon \Lambda_{0, q}\left(f_{i}^{*}\right), \quad i=1,2 .
$$

If (49) holds then using Lemma 7 and Remark 2 we obtain

$$
f_{i}^{2 p, q}(x) \leq c s_{D_{2 p}}(x) \Lambda_{0, p}\left(f_{i}^{2 p, q *}\right) \leq c s_{D_{2 p}}(x) \Lambda_{0, p, q}\left(f_{i}^{*}\right) \leq c \varepsilon s_{D_{2 p}}(x) \Lambda_{0, q}\left(f_{i}^{*}\right), \quad x \in D_{p} .
$$

Recall that $f_{i}=f_{i}^{2 p, q}+\widetilde{f}_{i}^{2 p, q}$. Thus, if (49) holds then we have

$$
\frac{(1+\varepsilon)^{-d-\alpha} \Lambda_{0, q}\left(f_{1}^{*}\right)}{\left(c \varepsilon+(1+\varepsilon)^{d+\alpha}\right) \Lambda_{0, q}\left(f_{2}{ }^{*}\right)} \leq \frac{f_{1}(x)}{f_{2}(x)} \leq \frac{\left(c \varepsilon+(1+\varepsilon)^{d+\alpha}\right) \Lambda_{0, q}\left(f_{1}^{*}\right)}{(1+\varepsilon)^{-d-\alpha} \Lambda_{0, q}\left(f_{2}{ }^{*}\right)}, \quad x \in D_{p},
$$

and, finally,

$$
\mathrm{RO}_{D_{p}} \frac{f_{1}}{f_{2}} \leq\left(c \varepsilon+(1+\varepsilon)^{d+\alpha}\right)^{2}(1+\varepsilon)^{2 d+2 \alpha} .
$$

We are satisfied with (51) for the moment. Let $0<\bar{p}<\bar{q} / 4<\bar{q}<1 / 2, g=f_{1}^{2 \bar{p}, \bar{q}}-m_{\bar{q}} f_{2}^{2 \bar{p}, \bar{q}}$, and $h=M_{\bar{q}} f_{2}^{2 \bar{p}, \bar{q}}-f_{1}^{2 \bar{p}, \bar{q}}$. Note that on $D_{2 \bar{p}}$ both $g$ and $h$ are Poisson integrals of nonnegative measures. If $D_{\bar{p}} \neq \emptyset$ then by Theorem 1 (see also (44)),

$$
\sup _{D_{\bar{p}}} \frac{f_{1}^{2 \bar{p}, \bar{q}}}{f_{2}^{2 \bar{p}, \bar{q}}}-m_{\bar{q}}=\sup _{D_{\bar{p}}} \frac{g}{f_{2}^{2 \bar{p}, \bar{q}}} \leq c^{4} \inf _{D_{\bar{p}}} \frac{g}{f_{2}^{22 \bar{p}, \bar{q}}}=c^{4}\left(\inf _{D_{\bar{p}}} \frac{f_{1}^{2 \bar{p}, \bar{q}}}{f_{2}^{2 \bar{p}, \bar{q}}}-m_{\bar{q}}\right),
$$

and

$$
M_{\bar{q}}-\inf _{D_{\bar{p}}} \frac{f_{1}^{2 \bar{p}, \bar{q}}}{f_{2}^{2 \bar{p}, \bar{q}}}=\sup _{D_{\bar{p}}} \frac{h}{f_{2}^{2 \bar{p}, \bar{q}}} \leq c^{4} \inf _{D_{\bar{p}}} \frac{h}{f_{2}^{2 \bar{p}, \bar{q}}}=c^{4}\left(M_{\bar{q}}-\sup _{D_{\bar{p}}} \frac{f_{1}^{2 \bar{p}, \bar{q}}}{f_{2}^{2 \bar{p}, \bar{q}}}\right) .
$$

By adding these inequalities we obtain

$$
\left(c^{4}+1\right)\left(\sup _{D_{\bar{p}}} \frac{f_{1}^{2 \bar{p}, \bar{q}}}{f_{2}^{22 \bar{p}, \bar{q}}}-\inf _{D_{\bar{p}}} \frac{f_{1}^{2 \bar{p}, \bar{q}}}{f_{2}^{2 \bar{p}, \bar{q}}}\right) \leq\left(c^{4}-1\right)\left(M_{\bar{q}}-m_{\bar{q}}\right) .
$$

We will now examine consequences of the following assumption:

$$
\Lambda_{0, \bar{q}}\left(f_{i}^{*}\right) \leq \varepsilon \Lambda_{0,2 \bar{p}, \bar{q}}\left(f_{i}^{*}\right) .
$$


By Lemma 7 and Remark 2 (consider $D=D_{2 \bar{p}}, p=2 \bar{p}$ and $B_{\bar{q}}$ replacing $B$ therein), for $x \in D_{\bar{p}}$ we have

$$
\widetilde{f}_{i}^{2 \bar{p}, \bar{q}}(x) \leq c s_{D_{2 \bar{p}}}(x) \Lambda_{0,2 \bar{p}}\left(\widetilde{f}_{i}^{2 \bar{p}, \bar{q} *}\right)=c s_{D_{2 \bar{p}}}(x) \Lambda_{0, \bar{q} / 2}\left(\widetilde{f}_{i}^{2 \bar{p}, \bar{q} *}\right) \leq c s_{D_{2 \bar{p}}}(x) \Lambda_{0, \bar{q}}\left(f_{i}^{*}\right) .
$$

Hence, by our assumption (53), Lemma 7 and Remark 2 applied to $D_{\bar{p}} \subset B_{2 \bar{p}}$

$$
\widetilde{f}_{i}^{2 \bar{p}, \bar{q}}(x) \leq c \varepsilon s_{D_{2 \bar{p}}}(x) \Lambda_{0,2 \bar{p}, \bar{q}}\left(f_{i}^{*}\right) \leq c \varepsilon s_{D_{2 \bar{p}}}(x) \Lambda_{0, \bar{p}}\left(f_{i}^{2 \bar{p}, \bar{q} *}\right) \leq c^{2} \varepsilon f_{i}^{2 \bar{p}, \bar{q}}(x), \quad x \in D_{\bar{p}} .
$$

Since $f_{i}=f_{i}^{2 \bar{p}, \bar{q}}+\widetilde{f}_{i}^{2 \bar{p}, \bar{q}}$ on $D_{2 \bar{p}}$, this and (52) yield

$$
\left(c^{4}+1\right)\left(M_{\bar{p}} /\left(1+c^{2} \varepsilon\right)-m_{\bar{p}}\left(1+c^{2} \varepsilon\right)\right) \leq\left(c^{4}-1\right)\left(M_{\bar{q}}-m_{\bar{q}}\right) .
$$

Note that $m_{\bar{p}} \geq m_{\bar{q}}$. Dividing by $m_{\bar{q}}$ finally gives

$$
\mathrm{RO}_{D_{\bar{p}}} \frac{f_{1}}{f_{2}} \leq\left(1+c^{2} \varepsilon\right)^{2}+\left(1+c^{2} \varepsilon\right) \frac{c^{4}-1}{c^{4}+1}\left(\mathrm{RO}_{D_{\bar{q}}} \frac{f_{1}}{f_{2}}-1\right) .
$$

We now come to the conclusion of our considerations. Let $\eta>0$. If $\varepsilon$ is small enough then the right hand side of (51) is smaller than $1+\eta$ and right hand side of (54) does not exceed $\varphi\left(\mathrm{RO}_{D_{\bar{q}}}\left(f_{1} / f_{2}\right)\right)$, where

$$
\varphi(t)=1+\frac{\eta}{2}+\frac{c^{4}}{c^{4}+1}(t-1), \quad t \geq 1 .
$$

Let $\varphi^{1}=\varphi, \varphi^{l+1}=\varphi\left(\varphi^{l}\right), l=1,2, \ldots$. Observe that $\varphi(t)=t$ for $t=1+\eta\left(c^{4}+1\right) / 2$, and $\varphi(t)<t$ for $t>1+\eta\left(c^{4}+1\right) / 2$. Thus the $l$-fold compositions $\varphi^{l}\left(c^{4}\right)$ converge to $1+\eta\left(c^{4}+1\right) / 2$ as $l \rightarrow \infty$. In what follows let $l$ be such that

$$
\varphi^{l}\left(c^{4}\right)<1+\eta\left(c^{4}+1\right) .
$$

Let $k$ be the least integer such that $k-1>c^{2} / \varepsilon^{2}$. We denote $n=l k$. Let $q_{0}=1 / 2$, $q_{j+1}=p\left(q_{j}\right)$ for $j=0, \ldots, n-1$ (see (48)), and $r=q_{n}$. If for any $j<n,(49)$ holds with $q=q_{j}$ and $p=p(q)=q_{j+1}$, then

$$
\mathrm{RO}_{D_{r}} \frac{f_{1}}{f_{2}} \leq \mathrm{RO}_{D_{q_{j+1}}} \frac{f_{1}}{f_{2}} \leq 1+\eta,
$$

and we are done by the definition of $\varepsilon$ and (50). Otherwise for $j=0, \ldots, n-1$, we have $\Lambda_{0, q_{j+1}, q_{j}}\left(f_{i}{ }^{*}\right)>\varepsilon \Lambda_{0, q_{j}}\left(f_{i}{ }^{*}\right)$ for $i=1$ or $i=2$. Note that by Lemma 7

$$
c^{-1} \frac{f_{i}(x)}{\Lambda_{0, q_{j}}\left(f_{i}^{*}\right)} \leq s_{D_{2 q_{j}}}(x) \leq c \frac{f_{3-i}(x)}{\Lambda_{0, q_{j}}\left(f_{3-i}{ }^{*}\right)}, \quad x \in D_{q_{j+1}, q_{j}} .
$$

Hence $\Lambda_{0, q_{j+1}, q_{j}}\left(f_{i}^{*}\right) / \Lambda_{0, q_{j}}\left(f_{i}^{*}\right) \leq c^{2} \Lambda_{0, q_{j+1}, q_{j}}\left(f_{3-i}{ }^{*}\right) / \Lambda_{0, q_{j}}\left(f_{3-i}{ }^{*}\right)$, and so $\Lambda_{0, q_{j+1}, q_{j}}\left(f_{i}{ }^{*}\right)>$ $c^{-2} \varepsilon \Lambda_{0, q_{j}}\left(f_{i}^{*}\right)$ for both $i=1$ and $i=2$ (and all $j=0,1, \ldots, n-1$ ). If $0 \leq j<l$ and $\bar{p}=q_{(j+1) k}, \bar{q}=q_{j k}$, then

$$
\Lambda_{0,2 \bar{p}, \bar{q}}\left(f_{i}^{*}\right) \geq \Lambda_{0, q_{(j+1) k-1}, q_{j k}}\left(f_{i}^{*}\right) \geq(k-1) c^{-2} \varepsilon \Lambda_{0, \bar{q}}\left(f_{i}^{*}\right) \geq \varepsilon^{-1} \Lambda_{0, \bar{q}}\left(f_{i}^{*}\right),
$$


so that (53) is satisfied. We conclude that (54) holds. Recall that $\mathrm{RO}_{D_{1 / 2}}\left(f_{1} / f_{2}\right) \leq c^{4}$. By the definition of $l$ and monotonicity of $\varphi$

$$
\operatorname{RO}_{D_{q_{l k}}} \frac{f_{1}}{f_{2}} \leq \varphi\left(\operatorname{RO}_{D_{q_{(l-1) k}}} \frac{f_{1}}{f_{2}}\right) \leq \ldots \leq \varphi^{l}\left(\operatorname{RO}_{D_{q_{0}}} \frac{f_{1}}{f_{2}}\right) \leq 1+\eta\left(c^{4}+1\right)
$$

i.e. $\mathrm{RO}_{D_{r}}\left(f_{1} / f_{2}\right) \leq 1+\eta\left(c^{4}+1\right)$. Since $\eta>0$ was arbitrary, the proof is complete.

Corollary 1 If $D$ is bounded, $0 \in \partial D$ and $y \in D^{c} \backslash\{0\}$, then

$$
\lim _{D \ni x \rightarrow 0} \frac{P_{D}(x, y)}{s_{D}(x)} \text { exists. }
$$

Proof: We may assume that $P_{D}(x, y)<\infty$ for $x \in D$. For bounded $D$, by (2) and (5) we have that

$$
s_{D}(x)=\lim _{|z| \rightarrow \infty} P_{D}(x, z) / \nu(0, z) .
$$

In fact, $P_{D}(x, z) /\left(s_{D}(x) \nu(0, z)\right) \rightarrow 1$ uniformly in $x \in D$. By scaling, we may assume that $|y| \geq 1$. We apply Lemma 8 to $\lambda_{1}=\varepsilon_{y}$ and $\lambda_{2}=\varepsilon_{z} / \nu(0, z)$. It follows that $\mathrm{RO}_{D_{r}} P_{D}(\cdot, y) / s_{D}(\cdot) \rightarrow 1$ as $r \rightarrow 0^{+}$, which, in presence of $(39)$, is equivalent to the convergence to a finite, positive limit.

As an addition to Corollary 1 we note that if 0 is inaccessible from $D$, then we have

$$
\lim _{D \ni x \rightarrow 0} \frac{P_{D}\left[\lambda_{1}\right](x)}{P_{D}\left[\lambda_{2}\right](x)}=\frac{\int_{\mathbf{R}^{d}} \nu(0, y) P_{D}^{*}\left[\lambda_{1}\right](d y)}{\int_{\mathbf{R}^{d}} \nu(0, y) P_{D}^{*}\left[\lambda_{2}\right](d y)},
$$

and

$$
\lim _{D \ni x \rightarrow 0} \frac{P_{D}\left[\lambda_{1}\right](x)}{s_{D}(x)}=\frac{\int_{\mathbf{R}^{d}} \nu(0, y) P_{D}^{*}\left[\lambda_{1}\right](d y)}{\int_{\mathbf{R}^{d}} \nu(0, y) s_{D}(y) d y+1} .
$$

Here $\lambda_{1}, \lambda_{2}$ are nonnegative measures on $B^{c}$ for which the Poisson integrals are positive and finite. Indeed, by Lemma 7 the integrals $\int \nu(0, y) P_{D}^{*}\left[\lambda_{i}\right](d y)$ are finite. Hence, for every $\varepsilon>0$ we can find $q>0$ such that (49) is satisfied with $p=q / 2$. It follows that (50) holds. Since $\varepsilon$ was arbitrary, the first equality is proved. The second one follows by using (56).

We remark in passing that (56) yields (24) as a consequence of (3), and sheds some light on the role of $s_{D}$ as a substitute, at infinity, for the Poisson kernel, see Theorem 2.

\section{$5 \quad$ Harmonicity}

Definition 1 Let $f$ be a nonnegative continuous function on $D$ and let $\lambda$ be a nonnegative measure on $D^{c}$. We say that $f$ is $\alpha$-harmonic in $D$ with outer charge $\lambda$ on $D^{c}$ if for every open bounded $U$ such that $\bar{U} \subset D$ we have (see (27))

$$
f(x)=\Omega_{U}^{D}[f, \lambda](x), \quad x \in U .
$$


We note that the integral in (59) is finite by the assumption that the left hand side of (59) is continuous (hence finite). Thus for (nonnegative) $f$ which is $\alpha$-harmonic on (nonempty open) $D$ with outer charge $\lambda \geq 0$, by (3) and (22) we necessarily have that

$$
\int_{D}(1+|y|)^{-d-\alpha} f(y) d y+\int_{D^{c}}(1+|y|)^{-d-\alpha} \lambda(d y)<\infty .
$$

The present definition extends the usual definition of an $\alpha$-harmonic function ([9]) by allowing measures as "boundary values" (outer charge) on $D^{c}$. The (genuine) $\alpha$-harmonic functions studied so far in the literature correspond to absolutely continuous measures $\lambda$. For such measures we can denote $d \lambda / d x$ by $f$ on $D^{c}$, and (59) then reads

$$
f(x)=\int_{U^{c}} f(y) \omega^{x}(d y), \quad x \in U,
$$

for open precompact $U \subset D$, see (21). We consider (59), (61) a mean-value property because $\omega_{U}^{x}$ is a probability measure.

Formula (26) yields that the function $x \mapsto G_{D}(x, y)$ is $\alpha$-harmonic on $D \backslash\{y\}$ with zero outer charge, see also Remark 1. Also, $x \mapsto \omega_{D}^{x}(A)$ is $\alpha$-harmonic on $D$ for every set $A$ by (18), and (21) applied to $U$. Here the outer charge is $\mathbf{1}_{A} d x$. Lastly, if $f=P_{D}(\cdot, y)$ is finite on $D$ then by (29) it is $\alpha$-harmonic in $D$ with outer charge $\varepsilon_{y}$. If a measure $\lambda$ is nonnegative and $P_{D}[\lambda]$ is finite on $D$ then by Fubini-Tonelli and Remark $1, P_{D}[\lambda]$ is $\alpha$ harmonic in $D$ with outer charge $\lambda$. By the same token, $x \mapsto P_{D}(x, y)$ is not $\alpha$-harmonic in $D$ with zero outer charge. To be absolutely clear on this, we consider $D=B$ and $|y|>1$. We note that the function $x \mapsto P_{B}(x, y)$ vanishes on $B^{c}$, and has a maximum inside $B$. Thus the mean-value property (59) cannot hold with $\lambda=0$ (recall that $\omega_{U}^{x}\left(\mathbf{R}^{d}\right)=1$ ). As we will see below, not every harmonic function is a Poisson integral, neither is every harmonic function on $D$ represented by an integral against the harmonic measure of $D$.

If $f$ is a function on $\mathbf{R}^{d}$ continuous on $D$ and $\lambda(d x)=f(x) d x$ on $D^{c}$, then (59) is equivalent to

$$
\Delta^{\alpha / 2} f(x)=0, \quad x \in D .
$$

The result is given in [13], and its proof can be extended to the present more general setting. However, we will not use (62) in the sequel, and we leave the verification of the extension to the interested reader. We also refer the reader to [16] to see the limitations of pointwise definition of harmonic functions by means of the corresponding generators.

Remark 8 If open precompact $D^{\prime} \subset D$ is a Lipschitz domain, then $\omega_{D^{\prime}}^{x}\left(\partial D^{\prime}\right)=0$ (see the discussion following (21)), and $f$ in Definition 1 can be considered a Poisson integral on $D^{\prime}$. This immediately yields Harnack inequality for general (nonnegative) harmonic functions, see (30), (31), and Remark 1.

In a number of considerations below we need to allow $U$ touching $\partial D$ in (59). Let $D^{(r)}$ be the set of regular boundary points for $D$ (see Section 2). It is known that $\omega_{U}^{x}\left(\partial D \backslash D^{(r)}\right)=0$ for every open $U$ and $x \in U$, and $\left|\partial D \backslash D^{(r)}\right|=0$ ([34]).

Lemma 9 Suppose that $0 \leq g \leq f$ on $D$, and $f, g$ are $\alpha$-harmonic on $D$ with zero outer charge. If $U \subset D$ and $f(x)=\int_{U^{c}} f(y) \omega_{U}^{x}(d y), x \in U$, then $g(x)=\int_{U^{c}} g(y) \omega_{U}^{x}(d y), x \in U$. 
Proof: Let $D_{n}$ be an increasing sequence of open sets precompact in $D$ such that $D=\bigcup D_{n}$. Let $U_{n}=U \cap D_{n}$, so that $U_{n}$ increase to (possibly unbounded) $U$. Then

$$
0 \leq \int_{U \backslash U_{n}} g(y) \omega_{U_{n}}^{x}(d y) \leq \int_{U \backslash U_{n}} f(y) \omega_{U_{n}}^{x}(d y)=f(x)-\int_{D \backslash U} f(y) \omega_{U_{n}}^{x}(d y), \quad x \in U_{n} .
$$

Recall that $\omega_{U_{n}}^{x}$ increase on $D \backslash U$ and vaguely converge to $\omega_{U}^{x}$ on $\mathbf{R}^{d}$. Since $D \backslash U \subset D$ and $\bigcap \overline{U \backslash U_{n}} \subset \partial D$ are disjoint, we conclude that actually $\omega_{U_{n}}^{x}$ increase to $\omega_{U}^{x}$ on $D \backslash U$. Hence, by monotone convergence, the right hand side tends to 0 when $n \rightarrow \infty$. Thus

$$
g(x)=\lim _{n \rightarrow \infty} \int_{D \backslash U_{n}} g(y) \omega_{U_{n}}^{x}(d y)=\lim _{n \rightarrow \infty} \int_{D \backslash U} g(y) \omega_{U_{n}}^{x}(d y)=\int_{D \backslash U} g(y) \omega_{U}^{x}(d y) .
$$

Lemma 10 Let $D_{1}, D_{2}$ be open sets such that

$$
\operatorname{dist}\left(D_{1} \backslash D_{2}, D_{2} \backslash D_{1}\right)>0 .
$$

Let $D=D_{1} \cup D_{2}$ and assume that $\omega_{D}^{x}\left(D^{c}\right)>0$ for (one and therefore for all) $x \in D$. Let $f \geq 0$ be a function on $\mathbf{R}^{d}$ such that $f=0$ on $D^{c}$, and for $i=1,2$ we have

$$
f(x)=\int f(y) \omega_{D_{i}}^{x}(d y) \quad \text { if } x \in D_{i} .
$$

If $D_{1}$ is bounded then $f=0$ on the whole of $D$.

Proof: Note that effectively the integration in (64) is only over $D_{3-i} \backslash D_{i}, i=1,2$. We can assume that both these sets are nonempty. We then observe that $f$ is bounded on $D_{1} \backslash D_{2}$. Indeed, if we consider Lipschitz open set $U$ such that $\overline{D_{1} \backslash D_{2}} \subset U \subset D_{1} \cup D^{c}$ and $\operatorname{dist}\left(U, D_{2} \backslash D_{1}\right)>0$ then it follows from (64) with $i=1,(18)$ and (22) that $f$ is a Poisson integral on $D_{1} \cap U$, and the boundedness follows from Remark 6. By considering $i=2$ in (64) we see that $f$ is bounded on $D_{2}$, hence on the whole of $D$.

Let $\left\{X_{t}, t \geq 0 ; \mathbf{P}_{x}, \mathbf{E}_{x}, x \in \mathbf{R}^{d}\right\}$ be the isotropic stable Lévy process with the corresponding Markov probabilities and expectations. For open set $U$ we define the first entrance time of $U^{c}, \tau_{U}=\inf \left\{t \geq 0: X_{t} \notin U\right\}$. It is well known that $\omega_{U}^{x}(A)=\mathbf{P}_{x}\left(\tau_{U}<\right.$ $\left.\infty, X_{\tau_{U}} \in A\right)$ for $x \in U$. Let $T_{1}=\tau_{D_{1}}$. We define "shuttle" times

$$
\begin{aligned}
T_{2 n} & =T_{2 n-1}+\tau_{D_{2}} \circ \Theta_{T_{2 n-1}}, \\
T_{2 n+1} & =T_{2 n}+\tau_{D_{1}} \circ \Theta_{T_{2 n}}, \quad n=1,2, \ldots,
\end{aligned}
$$

where $\Theta$ is the usual shift operator: $\left(X \circ \Theta_{s}\right)_{t}=X_{s+t}$. If $X_{T_{n}} \in D^{c}$ then

$$
\tau_{D}=T_{n}=T_{n+1}=T_{n+2}=\ldots, \quad \text { and } \quad X_{\tau_{D}}=X_{T_{n}}=X_{T_{n+1}}=\ldots
$$

Otherwise,

$$
\left|X_{T_{n+1}}-X_{T_{n}}\right| \geq \operatorname{dist}\left(\overline{D_{1} \backslash D_{2}}, \overline{D_{2} \backslash D_{1}}\right)=\operatorname{dist}\left(D_{1} \backslash D_{2}, D_{2} \backslash D_{1}\right)>0 .
$$

We define Markov time $T_{\infty}=\lim _{n \rightarrow \infty} T_{n}$. Clearly, $T_{\infty} \leq \tau_{D}$. By quasi-left-continuity of $\left\{X_{t}\right\}$ we have that $X_{T_{n}} \rightarrow X_{T_{\infty}}$ as $n \rightarrow \infty$, if $T_{\infty}<\infty$. But (66) then implies (65), in 
particular $T_{\infty}=\tau_{D}$. Clearly, if $T_{\infty}=\infty$, in particular if $T_{n}=\infty$ for some $n$, then we also have $T_{\infty}=\tau_{D}$. If $T_{n-1}=T_{n}<\infty$ then $X_{T_{n}} \in D^{c}$, and so by strong Markov property of $\left\{X_{t}\right\}$ and (64) we obtain

$$
\begin{aligned}
f(x) & =\mathbf{E}_{x}\left\{T_{n}<\infty ; f\left(X_{T_{n}}\right)\right\}=\mathbf{E}_{x}\left\{T_{n-1}<T_{n}<\tau_{D} ; f\left(X_{T_{n}}\right)\right\} \\
& \leq\|f\|_{\infty} \mathbf{P}_{x}\left\{T_{n-1}<T_{n}<\tau_{D}\right\}, \quad n \geq 2 .
\end{aligned}
$$

Here $\|f\|_{\infty}=\sup _{x \in \mathbf{R}^{d}} f(x)$. We consider

$$
\begin{aligned}
g_{n}(x) & =\mathbf{P}_{x}\left\{T_{n-1}<T_{n}<\tau_{D}\right\}=\mathbf{P}_{x}\left\{T_{n-3}<T_{n-2}<T_{n-1}<T_{n}<\tau_{D}\right\} \\
& =\mathbf{E}_{x}\left\{T_{n-3}<T_{n-2}<\tau_{D} ; \mathbf{P}_{X_{T_{n-2}}}\left[T_{1}<T_{2}<\tau_{D}\right]\right\} \\
& =\mathbf{E}_{x}\left\{T_{n-3}<T_{n-2}<\tau_{D} ; g_{2}\left(X_{T_{n-2}}\right)\right\} \leq\left\|g_{n-2}\right\|_{\infty}\left\|g_{2}\right\|_{\infty},
\end{aligned}
$$

where $n \geq 4$. We will examine

$$
\begin{aligned}
g_{2}(x) & =\mathbf{P}_{x}\left\{T_{1}<T_{2}<\tau_{D}\right\} \\
& =\mathbf{E}_{x}\left\{X_{\tau_{D_{1}}} \in D_{2} ; \mathbf{P}_{X_{\tau_{D_{1}}}}\left[\tau_{D_{2}}<\infty, X_{\tau_{D_{2}}} \in D_{1}\right]\right\} .
\end{aligned}
$$

If $g_{2} \equiv 1$ on $D$ then $g_{n} \equiv 1$ on $D$ for every even $n$, see (68). By the definition of $g_{n}$ and our discussion of (66), we then have that $\mathbf{P}_{x}\left\{\tau_{D}=\infty\right\}=1$, contradicting the assumptions of the lemma. Thus, $g_{2}(x)<1$ for some $x \in D$. Observing the expressions in (69), by Harnack inequality we conclude that

$$
\mathbf{P}_{x}\left\{X_{\tau_{D_{1}}} \in D_{2}\right\}<1 \text { for all } x \in D_{1}
$$

or

$$
\mathbf{P}_{x}\left\{\tau_{D_{2}}<\infty, X_{\tau_{D_{2}}} \in D_{1}\right\}<1 \text { for all } x \in D_{2} .
$$

Assume that (70) holds. By Theorem 4 applied to the above mentioned set $U \cap D_{1}$, there is $c>0$ such that

$$
\mathbf{P}_{x}\left\{X_{\tau_{D_{1}}} \in D_{2}\right\} \leq c \mathbf{P}_{x}\left\{X_{\tau_{D_{1}}} \notin D_{2}\right\}, \quad x \in D_{1} \backslash D_{2}
$$

thus

$$
\mathbf{P}_{x}\left\{X_{\tau_{D_{1}}} \in D_{2}\right\} \leq 1-c, \quad x \in D_{1} \backslash D_{2}
$$

and so $\left\|g_{2}\right\|_{\infty}<1-c$.

Assuming (71), there will be a ball $B^{\prime} \subset D_{2} \backslash D_{1}$ such that $\mathbf{E}\left\{X_{\tau_{D_{2}}} \in D_{1}\right\} \leq 1-\varepsilon$ for some $\varepsilon>0$. If (70) is false then $\mathbf{P}_{x}\left\{X_{\tau_{D_{1}}} \in D_{2}\right\}=1=\mathbf{P}_{x}\left\{X_{\tau_{D_{1}}} \in D_{2} \backslash D_{1}\right\}=$ $\mathbf{P}_{x}\left\{X_{\tau_{D_{1} \cup D^{c}}} \in D_{2} \backslash D_{1}\right\}$ for all $x \in D_{1}$. By (3) there is $c>0$, independent of $x \in D_{1} \backslash D_{2}$, such that $\mathbf{P}_{x}\left\{X_{\tau_{D_{1}}} \in B^{\prime}\right\} \geq \mathbf{P}_{x}\left\{X_{\tau_{B(x, r)}} \in B^{\prime}\right\} \geq c$, where $r=\operatorname{dist}\left(x, D_{2} \backslash D_{1}\right)$. We obtain that $\left\|g_{2}\right\|_{\infty} \leq 1-c \varepsilon$.

Thus, (70) and (71) imply that $\left\|g_{2 n}\right\|_{\infty} \rightarrow 0$, and so $f \equiv 0$, see (67), (68).

Lemma 10 applies, e.g., if $D_{1}, D_{2}$ are overlapping finite open intervals on the line. 
Lemma 11 Let $f$ be a nonnegative function on $\mathbf{R}^{d}$ which is $\alpha$-harmonic in bounded $D$, with outer charge $f(x) d x$. Suppose that $f$ is bounded and continuous on $D \cup D^{(r)}$. Then

$$
f(x)=\int f(y) \omega_{D}^{x}(d y), \quad x \in D .
$$

Proof: Let $D_{n}$ be an increasing sequence of open sets precompact in $D$ such that $\cup D_{n}=D$. Recall that $\omega_{D_{n}}^{x} \rightarrow \omega_{D}^{x}$ weakly as $n \rightarrow \infty$ and $\omega_{D}^{x}\left(\bar{D} \backslash D^{(r)}\right)=0$. Since $f$ is bounded and continuous on $D \cup D^{(r)}$,

$$
\lim _{n \rightarrow \infty} \int_{\bar{D}} f(y) \omega_{D_{n}}^{x}(d y)=\lim _{n \rightarrow \infty} \int_{D \cup D^{(r)}} f(y) \omega_{D_{n}}^{x}(d y)=\int_{D \cup D^{(r)}} f(y) \omega_{D}^{x}(d y)=\int_{\bar{D}} f(y) \omega_{D}^{x}(d y) .
$$

We obtain (73) by noting that $P_{D_{n}}(x, y) \nearrow P_{D}(x, y)$ for $x \in D, y \in D^{c}$, and so

$$
\lim _{n \rightarrow \infty} \int_{\bar{D}^{c}} P_{D_{n}}(x, y) f(y) d y=\int_{\bar{D}^{c}} P_{D}(x, y) f(y) d y .
$$

We remark in passing that (73) implies $\alpha$-harmonicity through (18). The reverse implication is not true as we will see from the example of the Martin kernel with the pole at an accessible boundary point.

\section{$6 \quad$ Martin kernel}

It follows from (60), (26) and (21) that for open Greenian $D$,

$$
\int_{\mathbf{R}^{d}} G_{D}(x, v)(1+|v|)^{-d-\alpha} d v<\infty, \quad x \in \mathbf{R}^{d} .
$$

By Lemma $7, s_{D \cap B(y, 1)}(v)$ is comparable to $G_{D}\left(x_{0}, v\right)$ at $v=y$, thus $(7)$ is equivalent to

$$
P_{D}\left(x_{0}, y\right)=\int_{\mathbf{R}^{d}} G_{D}\left(x_{0}, v\right) \nu(v, y) d v<\infty .
$$

Here $y \in \mathbf{R}^{d}$. If $D$ is non-Greenian, then $s_{D \cap B(y, 1)}=s_{B(y, 1)}$ for every $y \in \mathbf{R}^{d}$ because $D^{c}$ is polar, and so every point of $D^{c}$ is accessible from $D$. Thus, for general $D$,

$$
\partial_{M} D \cap \mathbf{R}^{d}=\left\{y \in \partial D: P_{D}\left(x_{0}, y\right)=\infty\right\} .
$$

By Fatou's lemma the function $y \mapsto \Lambda_{y}\left(G_{D}\left(x_{0}, \cdot\right)\right)$ is lower semicontinuous. We see that $\partial_{M} D$ is Borel measurable, and in fact of type $\mathcal{G}_{\delta}$.

Proof of Theorem 2: Let $D$ be open and Greenian, and let $y \in \partial D$. By translation invariance, to study $M_{D}(\cdot, y)$ we may assume with no loss of generality, that $y=0$.

Let $x \in D, \rho=\left(|x| \wedge\left|x_{0}\right|\right) / 2$, and $D_{\rho}=D \cap B(0, \rho)$. By Harnack inequality in the first variable, $G_{D}(x, v) / G_{D}\left(x_{0}, v\right)$ is bounded from above and below for $v \in D_{\rho}$. Also, $G_{D}(x, v)=P_{D_{\rho}}[G(x, u) d u](v), G_{D}\left(x_{0}, v\right)=P_{D_{\rho}}\left[G\left(x_{0}, u\right) d u\right](v)$ for $v \in D_{\rho}$. Lemma 8 , applied to $D_{\rho}$, yields that $M_{D}(x, 0)$ is well-defined by (8). Clearly, $0<M_{D}(x, 0)<\infty$. 
Denote $M(x)=M_{D}(x, 0)$. If 0 is inaccessible from $D$, then by $(57)$ we have

$$
M(x)=\int_{D} \nu(0, y) G_{D}(x, y) d y / \int_{D} \nu(0, y) G_{D}\left(x_{0}, y\right) d y=P_{D}(x, 0) / P_{D}\left(x_{0}, 0\right),
$$

in particular $M(x)$ is $\alpha$-harmonic on $D$ with outer charge $\left(P_{D}\left(x_{0}, 0\right)\right)^{-1} \varepsilon_{0}$ and so it is not $\alpha$-harmonic on $D$ with zero outer charge. However, if 0 is accessible from $D$ then

$$
M(x)=\int_{D \backslash U} M(y) \omega_{U}^{x}(d y), \quad x \in U
$$

for every $U=D \backslash \bar{B}_{R}$ with $R>0$. Indeed, (77) is equivalent to uniform integrability of $G_{D}(y, z) / G_{D}\left(x_{0}, z\right)$ with respect to $\omega_{U}^{x}(d y)$ on the (bounded) set $D \backslash U$ as $D \ni z \rightarrow 0$. To prove the uniform integrability, let $0<r<\min \left(R / 4,\left|x_{0}\right| / 4\right)$ and $z_{0} \in D_{r}$ be a fixed point. For $y \in D_{R} \backslash D_{3 r}$ and $z \in D_{r}$, Remark 7 yields that

$$
\frac{G_{D}(y, z)}{G_{D}\left(x_{0}, z\right)} \leq C_{d, \alpha, r} \frac{G_{D}\left(y, z_{0}\right)}{G_{D}\left(x_{0}, z_{0}\right)} .
$$

Again by Remark 7 we obtain that $\sup _{y \in D_{R} \backslash D_{3 r}} G_{D}\left(y, z_{0}\right)<\infty$. Thus we only need to estimate $\int_{D_{3 r}} G_{D}(z, y) \omega_{U}^{x}(d y) / G_{D}\left(x_{0}, z\right)$ for $z \in D_{r}$.

Since the density function (Poisson kernel) of $\omega_{U}^{x}$ is bounded on $D_{3 R / 4}$, we have

$$
\int_{D_{3 r}} G_{D}(y, z) \omega_{U}^{x}(d y) \leq C_{d, \alpha, D, R} \int_{D_{3 r}} G_{D}(y, z) d y .
$$

By (22), $\omega_{D \backslash D_{3 r}}^{x_{0}}$ is absolutely continuous on $\bar{D}_{3 r}$ with respect to the Lebesgue measure, and has $P_{D \backslash \bar{D}_{3 r}}\left(x_{0}, \cdot\right)$ as density function. Thus

$$
\begin{aligned}
G_{D}\left(x_{0}, z\right) & =\int_{D_{3 r}} G_{D}(y, z) P_{D \backslash \bar{D}_{3 r}}\left(x_{0}, y\right) d y \\
& =\int_{D_{3 r}} \int_{D \backslash \bar{D}_{3 r}} G_{D}(y, z) G_{D \backslash \bar{D}_{3 r}}\left(x_{0}, \zeta\right) \nu(\zeta, y) d \zeta d y \\
& \geq 2^{-d-\alpha}\left(\int_{D_{3 r}} G_{D}(y, z) d y\right)\left(\int_{D \backslash \bar{D}_{3 r}} G_{D \backslash \bar{D}_{3 r}}\left(x_{0}, \zeta\right) \nu(\zeta, 0) d \zeta\right) .
\end{aligned}
$$

The last integral becomes arbitrarily large when $r$ is small enough. This is because $\int_{D} G_{D}\left(x_{0}, \zeta\right) \nu(\zeta, 0) d \zeta=\infty, 0$ is accessible from $D$, and $G_{D}\left(x_{0}, \cdot\right) \approx s_{D \cap B}(\cdot)$ at 0 by Lemma 7.

Combining this, (79), and (78), we obtain the uniform integrability, and (77). In fact, (18) yields (77) for every open $U \subset D$ provided $0 \notin \bar{U}$. In particular, $M$ is $\alpha$-harmonic on $D$. Regarding the remark at the end of Section 5 we note that $f=M$ violates (73) because $M$ vanishes on $D^{c}$.

We now turn to the Martin kernel with the pole at infinity. Let $x \in D$. If $D=\mathbf{R}^{d}$ and $\mathbf{R}^{d}$ is Greenian, or $\alpha<d$, then $M_{D}(x, \infty)=\lim _{|v| \rightarrow \infty}|v-x|^{\alpha-d} /\left|v-x_{0}\right|^{\alpha-d}=1, s_{D} \equiv \infty$, and we are done. Without loosing generality we may suppose in what follows that $D$ is 
a proper unbounded (Greenian) subset of $\mathbf{R}^{d}$, and $0 \in D^{c}$. Consider the inversion with respect to the unit sphere:

$$
T x=\frac{1}{|x|^{2}} x, \quad x \neq 0 .
$$

Inversion can be used to reduce potential theoretic problems for the point at infinity to those at 0 , see [17] for a detailed discussion. As proved in [17], the set $T D=\{T x: x \in D\}$ has Green function

$$
G_{D}(x, v)=|x|^{\alpha-d}|v|^{\alpha-d} G_{T D}(T x, T v), \quad x, v \neq 0,
$$

in particular it is Greenian. We obtain

$$
M_{D}(x, \infty)=\lim _{D \ni v \rightarrow \infty} \frac{|x|^{\alpha-d}|v|^{\alpha-d} G_{T D}(T x, T v)}{\left|x_{0}\right|^{\alpha-d}|v|^{\alpha-d} G_{T D}\left(T x_{0}, T v\right)}=\frac{|x|^{\alpha-d}}{\left|x_{0}\right|^{\alpha-d}} M_{T D}(T x, 0) .
$$

Here $M_{T D}$ denotes the Martin kernel of $T D$ with the pole at 0 and the reference point at $T x_{0}$. The existence of $M_{D}(x, \infty)$ defined by (8) is proved. Also, $0<M_{D}(x, \infty)<\infty$. Note that $|x|^{\alpha-d} M_{T D}(T x, 0)$ in (81) is the Kelvin transform of $M_{T D}(x, 0)$, see [17]. By [17], $M_{D}(x, \infty)$ is $\alpha$-harmonic in $D$ if and only if $M_{T D}(x, 0)$ is $\alpha$-harmonic in $T D$. We finally observe that $\infty$ is accessible for $D$ if and only if 0 is accessible for $T D$. Indeed, by (80) and a change of variable $v=T y$ with Jacobian $|y|^{-2 d}$,

$$
\int G_{T D}(T x, y) \nu(0, y) d y=\mathcal{A}_{d,-\alpha}|x|^{d-\alpha} \int G_{D}(x, T y)|y|^{-2 d} d y=\mathcal{A}_{d,-\alpha}|x|^{d-\alpha} s_{D}(x) .
$$

Therefore $\alpha$-harmonicity of $M_{D}(x, \infty)$ is equivalent to accessibility of $\infty$ for $D$.

We let

$$
M_{D}(x, y)=\frac{G_{D}(x, y)}{G_{D}\left(x_{0}, y\right)}, \quad x, y \in D
$$

so that $M_{D}(x, y)$ is now defined for all $x \in D$ and $y \in D^{*}$. In passing we note that $(80)$ yields

$$
M_{D}(x, y)=\frac{|x|^{\alpha-d}}{\left|x_{0}\right|^{\alpha-d}} M_{T D}(T x, T y), \quad x \in D, \quad y \in D^{*}
$$

where $0 \notin D$, the reference point for $M_{T D}$ is $T x_{0}$ and we use the convention $T 0=\infty$, $T \infty=0$. Recall that $B_{r}=B(0, r)$.

Lemma 12 For every $\rho>0$ and $\eta>0$ there is $r>0$ such that for every Greenian $D$

$$
\begin{gathered}
\mathrm{RO}_{y \in \bar{D} \cap B_{r}} M_{D}(x, y) \leq 1+\eta, \quad \text { if } \quad x, x_{0} \in D \backslash \bar{B}_{\rho}, \\
\mathrm{RO}_{y \in D^{*} \backslash \bar{B}_{1 / r}} M_{D}(x, y) \leq 1+\eta, \quad \text { if } \quad x, x_{0} \in D \cap \bar{B}_{1 / \rho} .
\end{gathered}
$$

The Martin kernel $M_{D}(x, y): D \times D^{*} \backslash\left\{\left(x_{0}, x_{0}\right)\right\} \mapsto[0, \infty]$ is jointly continuous.

Proof: To prove (85) let $\rho>r>0$ and $x, x_{0} \in D \backslash \bar{B}_{\rho}$. We note that by (8)

$$
\sup _{y \in \bar{D} \cap B_{r}} M_{D}(x, y)=\sup _{y \in D \cap B_{r}} \frac{G_{D}(x, y)}{G_{D}\left(x_{0}, y\right)}, \quad \inf _{y \in \bar{D} \cap B_{r}} M_{D}(x, y)=\inf _{y \in D \cap B_{r}} \frac{G_{D}(x, y)}{G_{D}\left(x_{0}, y\right)},
$$


hence $\mathrm{RO}_{y \in \bar{D} \cap B_{r}} M_{D}(x, y)=\mathrm{RO}_{y \in D \cap B_{r}} M_{D}(x, y)$. As functions of $y, G_{D}(x, y)$ and $G_{D}\left(x_{0}, y\right)$ are nonnegative Poisson integrals on $D \cap B_{\rho}$ of measures on $B_{\rho}^{c}$. Thus (85) is an immediate consequence of Lemma 8 and scaling. To prove (86), as in the proof of Theorem 2 we may assume that $0 \in D^{c}$, and then (81) reduces (86) to (85) for $T D$.

By Lemma $4, M_{D}$ given by (83) is jointly continuous: $D \times D \backslash\left\{\left(x_{0}, x_{0}\right)\right\} \mapsto[0, \infty]$. We will consider the remaining case; let $D \times D^{*} \ni\left(x^{\prime}, y^{\prime}\right) \rightarrow(x, y) \in D \times \partial_{*} D$. We have

$$
\frac{M_{D}\left(x^{\prime}, y^{\prime}\right)}{M_{D}(x, y)}=\frac{M_{D}\left(x^{\prime}, y^{\prime}\right)}{M_{D}\left(x, y^{\prime}\right)} \cdot \frac{M_{D}\left(x, y^{\prime}\right)}{M_{D}(x, y)} .
$$

Here the second factor on the right hand side converges to 1 by (85) or (86). We will verify uniform continuity of the first factor at $x^{\prime}=x$. If $\bar{B}(x, s) \subset D$, and $y^{\prime} \in \bar{B}(x, s)^{c}$ then by (26), (77) and (29) we see that $f(\cdot)=M_{D}\left(\cdot, y^{\prime}\right)$ satisfies (59) with $\lambda(d w)=$ $M_{D}\left(w, y^{\prime}\right) d w+c \varepsilon_{y^{\prime}}(d w)$ and $U$ being the open ball. Here $c=1 / P_{D}\left(x_{0}, y^{\prime}\right)$ if $y^{\prime} \in D^{c}$ and $y^{\prime}$ is inaccessible for $D$, and $c=0$ otherwise. The uniform continuity follows from (27) as in the proof of Lemma 4 , and $M_{D}\left(x^{\prime}, y^{\prime}\right) / M_{D}(x, y) \rightarrow 1$.

Remark 9 The kernel functions $G_{D}, P_{D}$, and $M_{D}$ may be studied without explicit mention of harmonicity, by using (15) and its consequences, (29) and (77).

\section{Structure of nonnegative harmonic functions}

Lemma 13 If $f \geq 0$ and $f$ is $\alpha$-harmonic on $D$, with outer charge $\lambda \geq 0$, then there is a unique nonnegative function $f_{s} \alpha$-harmonic in $D$ with zero outer charge such that $f=P_{D}[\lambda]+f_{s}$ on $D$.

Proof: Let $D_{n}$ be an increasing sequence of open precompact subsets of $D$ such that $\bigcup_{n=1}^{\infty} D_{n}=D$. By $(2)$, monotone convergence of $G_{D_{n}}$ to $G_{D}$, $\alpha$-harmonicity of $f$, and (21), we have

$$
P_{D}[\lambda](x)=\lim _{n \rightarrow \infty} \int_{D^{c}} \int_{D} G_{D_{n}}(x, z) \nu(z, y) d z \lambda(d y) \leq f(x), \quad x \in D .
$$

We let $f_{s}=f-P_{D}[\lambda]$. The stated properties easily follow.

Since the outer charge of $f_{s}$ vanishes on $D^{c}$, the present setting for further decomposition of $f_{s}$ is analogous to those of $[10,36,41]$, despite the initial generality of our definition of harmonic functions (but see the discussion following (9)).

Lemma 14 Let $D$ be Greenian and let $\mu \geq 0$ be a finite measure on $\partial_{M} D$. Then

$$
f(x)=\int_{\partial_{M} D} M_{D}(x, y) \mu(d y), \quad x \in \mathbf{R}^{d}
$$

is $\alpha$-harmonic on $D$ with zero outer charge. Conversely, if $f \geq 0$ is $\alpha$-harmonic on $D$ with zero outer charge then there is a unique finite measure $\mu \geq 0$ on $\partial_{M} D$ satisfying (87). 
ProOF: It is a straightforward consequence of Theorem 2 that $f$ given by $(87)$ is $\alpha$ harmonic in $D$ with zero outer charge on $D^{c}$. We shall write $f=M_{D}[\mu]$.

Let $f$ be a nonnegative function $\alpha$-harmonic in $D$ with zero outer charge on $D^{c}$. Let $D_{n}$ denote an increasing sequence of open sets precompact in $D$ such that $\bigcup_{n=1}^{\infty} D_{n}=D$. We will also assume that $\omega_{D_{n}}^{x}\left(\partial D_{n}\right)=0$ for $x \in D_{n}$, which holds, e.g., if $D_{n}$ are Lipschitz domains. By (22) for $x \in D_{n}$ we then have

$$
f(x)=\int_{D \backslash D_{n}} P_{D_{n}}(x, y) f(y) d y=\int_{D_{n}} M_{D_{n}}(x, v)\left(G_{D_{n}}\left(x_{0}, v\right) \int_{D \backslash D_{n}} \nu(v, y) f(y) d y\right) d v .
$$

Let $\mu_{n}(d v)=\left(G_{D_{n}}\left(x_{0}, v\right) \int_{D \backslash D_{n}} \nu(v, y) f(y) d y\right) d v$. Since $\mu_{n}(D)=f\left(x_{0}\right)<\infty$, by considering a subsequence we may assume that $\mu_{n}$ weakly converge to a finite nonnegative measure $\mu$ on $D^{*}$. We claim that $\mu$ is supported in $\partial_{*} D$. Indeed, if $n>k, v \in D_{k}$, $y \in D \backslash D_{n}$, then $\nu(v, y) \leq C_{k}$ and $G_{D_{n}}\left(x_{0}, v\right) \leq G_{D}\left(x_{0}, v\right)$. Hence

$$
\mu_{n}\left(D_{k}\right) \leq C_{k}\left(\int_{D_{k}} G_{D}\left(x_{0}, v\right) d v\right)\left(\int_{D \backslash D_{n}} f(y)(1+|y|)^{-d-\alpha} d y\right) \rightarrow 0
$$

as $n \rightarrow \infty$, see (60). This proves that $\mu\left(D_{k}\right)=0$ and so $\mu$ is a measure on $\partial_{*} D$.

Let $\varepsilon>0$ and $x \in D$. By Lemma 12 for every $y \in \partial_{*} D$ there exists its neighborhood $V_{y}$ such that

$$
\mathrm{RO}_{V_{y}} M_{U}(x, \cdot) \leq 1+\varepsilon,
$$

with $U=D$ and $U=D_{n}, n=1, \ldots$ From these, one selects a finite family $\left\{V_{j}, j=\right.$ $1, \ldots, m\}$ such that $V=V_{1} \cup \ldots \cup V_{m} \supset \partial_{*} D$. For $j=1, \ldots, m$, let $z_{j} \in V_{j} \cap D$. Let $k$ be so large that for $n \geq k$ we have $z_{j} \in D_{n}$, and

$$
(1+\varepsilon)^{-1} \leq \frac{M_{D}\left(x, z_{j}\right)}{M_{D_{n}}\left(x, z_{j}\right)} \leq 1+\varepsilon, \quad j=1, \ldots, m .
$$

If $v \in V_{j} \cap D_{n}$ then

$$
(1+\varepsilon)^{-3} \leq \frac{M_{D}(x, v)}{M_{D}\left(x, z_{j}\right)} \cdot \frac{M_{D}\left(x, z_{j}\right)}{M_{D_{n}}\left(x, z_{j}\right)} \cdot \frac{M_{D_{n}}\left(x, z_{j}\right)}{M_{D_{n}}(x, v)} \leq(1+\varepsilon)^{3} .
$$

Therefore

$$
(1+\varepsilon)^{-3} \leq \frac{\int_{D \cap V} M_{D}(x, y) \mu_{n}(d y)}{\int_{D \cap V} M_{D_{n}}(x, y) \mu_{n}(d y)} \leq(1+\varepsilon)^{3}, \quad n \geq k .
$$

By letting $n \rightarrow \infty$ we obtain

$$
(1+\varepsilon)^{-3} \leq \frac{\int_{\partial_{*} D} M_{D}(x, y) \mu(d y)}{f(x)} \leq(1+\varepsilon)^{3},
$$

which gives $f(x)=\int_{\partial_{*} D} M_{D}(x, y) \mu(d y)$.

We will prove that $\mu$ is concentrated on $\partial_{M} D$. Let $U$ be open and precompact in $D$ and let $x \in U$. By Theorem 2 and (29), if $y \in \partial_{*} D$, then $M_{D}(x, y) \geq \int_{D \backslash U} M_{D}(z, y) \omega_{U}^{x}(d z)$ and equality holds if and only if $y \in \partial_{M} D$. By Fubini's theorem

$$
0=f(x)-\int_{D \backslash U} f(z) \omega_{U}^{x}(d z)=\int_{\partial D}\left(M_{D}(x, y)-\int_{D \backslash U} M_{D}(z, y) \omega_{U}^{x}(d z)\right) \mu(d y),
$$


hence $\mu\left(\partial_{*} D \backslash \partial_{M} D\right)=0$.

We will prove the uniqueness of $\mu$ in the representation (87). We first consider $f=$ $M_{D}\left[\varepsilon_{y_{0}}\right]=M_{D}\left(\cdot, y_{0}\right)$, where $y_{0} \in \partial_{M} D$. To simplify notation, we assume as we may that $y_{0}=0$ (we use translation invariance if $0 \neq y_{0} \in \mathbf{R}^{d}$ and inversion if $y_{0}=\infty$ ).

Let $D_{r}=D \cap B_{r}, D_{r}^{\prime}=D \backslash \bar{B}_{r}$. Suppose that $f$ satisfies (87) for a nonnegative measure $\mu$ on $\partial_{M} D$. Let $r>0$ and $g(x)=\int_{|y|>3 r} M_{D}(x, y) \mu(d y)$. Considering $y \in \partial_{M} D$ such that $|y|>3 r$, by (77) we get

$$
g(x)=\int_{D \backslash D_{2 r}} g(z) \omega_{D_{2 r}}^{x}(d z), \quad x \in D_{2 r} .
$$

On the other hand, we may apply Lemma 9 to $f, g$, and $D_{r}^{\prime}$, to verify that

$$
g(x)=\int_{D \backslash D_{r}^{\prime}} g(z) \omega_{D_{r}^{\prime}}^{x}(d z), \quad x \in D_{r}^{\prime} .
$$

Lemma 10 yields $g(x)=\int_{D^{c}} g(z) \omega_{D}^{x}(d z)=0$, that is, $\mu=0$ on $\partial_{M} D \cap\{|y|>3 r\}$. In particular, the measures $\mu_{n}$ considered at the beginning of the proof, corresponding to $f(\cdot)=M_{D}\left(\cdot, y_{0}\right)$, weakly converge to $\varepsilon_{y_{0}}$. Fubini's theorem and dominated convergence yield that for general $f=M_{D}[\mu]$ the measures $\mu_{n}$ corresponding to $f$ weakly converge to $\mu$. Since $\mu_{n}$ are determined by $f$, so is $\mu$.

We note that if $f$ is $\alpha$-harmonic in $D$ (with zero outer charge) and $0 \leq f \leq M_{D}\left(\cdot, y_{0}\right)$ then the proof of Lemma 14 yields $f=c M_{D}\left(\cdot, y_{0}\right)$ for some $c \in[0,1]$. Thus, $M_{D}\left(\cdot, y_{0}\right)$ is minimal harmonic i.e. an extremal point of the class of nonnegative functions $f \alpha$ harmonic on $D$ (with zero outer charge), such that $f\left(x_{0}\right)=1$. We note, however, that our proof of Lemma 14 does not invoke Choquet's theorem. Instead it relies on (8) and Lemma 8.

Proof of Theorem 3: The theorem collects results of Lemma 13 and 14.

In particular, if the point at infinity is inaccessible for $D$ then $\infty$ is not charged by the measure $\mu$ in the representation $(87)$, and $M(\cdot, \infty)=s_{D}$ is not $\alpha$-harmonic in $D$ with zero outer charge, compare (16).

\section{Miscelanea}

Consider $f(x)=\omega_{D}^{x}\left(\partial_{M} D\right), x \in \mathbf{R}^{d}$. By Lemma 13,

$$
\int_{\partial_{M} D} P_{D}(x, y) d y \leq f(x) \leq 1, \quad x \in D
$$

Since $P_{D}(x, y)=\infty$ for $y \in \partial_{M} D$, we conclude that $\left|\partial_{M} D\right|=0$.

We will now strengthen the result of Lemma 1 and (22).

Proposition 1 For Greenian $D \subset \mathbf{R}^{d}$, and $x \in D$, the harmonic measure $\omega_{D}^{x}$ is absolutely continuous on $D^{c} \backslash \partial_{M} D$ with respect to the Lebesgue measure, with density $P_{D}(x, \cdot)$. 
Proof: Let $K \subset D^{c} \backslash \partial_{M} D$ be compact and let $f(x)=\omega_{D}^{x}(K)-P_{D}\left[\mathbf{1}_{K}\right](x) \geq 0$. We will verify that $f=0$. By Theorem $3, f(x)=\int_{\partial_{M} D} M_{D}(x, y) \mu(d y)$ for some nonnegative finite measure $\mu$ on $\partial_{M} D$. Let $L \subset \partial_{M} D$ be compact and let $g(x)=\int_{L} M_{D}(x, y) \mu(d x)$. It suffices to prove that $g=0$. We let

$$
U=\{x \in D: 2 \operatorname{dist}(x, K) \leq \operatorname{dist}(x, L)\}, \quad V=\{x \in D: 2 \operatorname{dist}(x, L) \leq \operatorname{dist}(x, K)\} .
$$

Observe that by (77), $g(x)=\int_{D \backslash U} g(y) \omega_{U}^{x}(d y)$ for $x \in U$. On the other hand, Lemma 9 applied to $\omega_{D}^{x}(K), g$, and $V \subset D$ yields $g(x)=\int_{D \backslash V} g(y) \omega_{V}^{x}(d y)$ for $x \in V$. Hence we may apply Lemma 10 to conclude that $g(x)=0$.

In particular, if $f=P_{D}[\lambda]$, where $\lambda$ is nonnegative and absolutely continuous with respect to the Lebesgue measure on $D^{c}$ and has a density function $g$, then we can write

$$
f(x)=\int_{D^{c}} g(y) \omega_{D}^{x}(d y), \quad x \in D
$$

This, however, requires a convention that $g(y)=0$ for $y \in \partial_{M} D$ on the right hand side, and should be used with caution. Another common convention is writing $f$ instead of $g$ above, see (61).

We note that there are domains $D$ for which the part of the harmonic measure which is singular with respect to the Lebesgue measure (i.e. $\omega_{D}^{x}$ on $\partial_{M} D$ ) is positive. Indeed, such is the complement of every closed non-polar set of zero Lebesgue measure, for example, the complement of a point on the line if $1<\alpha<2$, see [38].

Lemma 15 Every nonnegative $f$ harmonic on non-Greenian D is constant on D.

Proof: If $\alpha<d$ then $G_{D}$ is majorized by the Riesz kernel [34] and so every (open) $D \subset \mathbf{R}^{d}$ is Greenian. For $\alpha \geq d=1$, by [38], if $D$ is non-Greenian then $D^{c}$ is polar. In this case, let $x, y \in D$ and $0<r<\min \left(\operatorname{dist}\left(y, D^{c}\right),|y-x|\right)$. By recurrence (see [38] for the definition) for every $\varepsilon>0$ there is an open precompact $B \subset D$ such that $x \in B$ and $\omega_{B \backslash \bar{B}(y, r)}^{x}(B(y, r))>1-\varepsilon$. Using small $\varepsilon$ and $r$, and continuity of $f$ at $y$ we obtain $f(x) \geq f(y)$, hence $f$ is constant on $D$.

We will give examples of accessible and inaccessible boundary points. Let $d \geq 2$ and let $f:(0,1) \rightarrow(0, \infty)$ be any bounded increasing function. We define a thorn $D_{f}$ by (cf. $[19]):$

$$
D_{f}=\left\{\left(x_{1}, \ldots, x_{d}\right) \in \mathbf{R}^{d}: 0<x_{1}<1,\left|\left(x_{2}, \ldots, x_{d}\right)\right|<f\left(x_{1}\right)\right\} .
$$

Proposition 2 The origin is inaccessible from $D_{f}$ if and only if $\int_{0}^{1} t^{-d-\alpha} f(t)^{d+\alpha-1} d t<$ $\infty$

Proof: We denote the above integral by $I_{f}$. We need to prove that $\Lambda_{0}\left(s_{D_{f}}\right)=\infty$ if and only if $I_{f}=\infty$. Let $g(t)=\frac{1}{2}\left(f(t / 2) \wedge t\right.$ ). Note that $I_{f}=\infty$ if and only if $I_{g}=\infty$ (see the proof of Theorem 1.1(i) in [19]). For small $x \in D_{g}$ we have $B\left(x, g\left(x_{1}\right)\right) \subset D_{f}$, hence

$$
s_{D_{f}}(x) \geq s_{B\left(x, g\left(x_{1}\right)\right)}(x)=C_{d, \alpha}\left(g\left(x_{1}\right)\right)^{\alpha} .
$$


Thus, if $I_{f}=\infty$ then $\left(I_{g}=\infty\right.$ and $) \Lambda_{0}\left(s_{D_{f}}\right)=\infty$. We may now assume that $I_{f}$ is finite and $f(t) \leq|t|$. Let $D_{f, r}=D_{f} \cap B_{r}$ for $r>0$. Let $r<1 / 4$. We have

$$
s_{D_{f}}(x)=s_{D_{f, 4 r}}(x)+\int_{D_{f} \backslash D_{f, 4 r}} s_{D_{f}}(y) \omega_{D_{f, 4 r}}^{x}(d y) .
$$

The latter term is a Poisson integral on $D_{f, 4 r}$. In view of Lemma 7

$$
s_{D_{f}}(x) \leq s_{D_{f, 4 r}}(x)\left(1+C_{d, \alpha} \Lambda_{0,3 r}\left(s_{D_{f}}\right)\right), \quad x \in D_{f, 3 r} .
$$

Let $M(r)=\sup _{x_{1}=r} s_{D_{f}}(x) /(f(4 r))^{\alpha}$. Inscribing $D_{f, r}$ into a cylinder and observing that $G_{D}(x, y) \leq C_{d, \alpha}|x-y|^{-d+\alpha}$ we can show that $s_{D_{f, r}}(x) \leq C_{d, \alpha}(f(r))^{\alpha}$. We thus obtain

$$
M(r) \leq c_{1}+c_{2} \int_{2 r}^{1} M(t)(f(4 t))^{d+\alpha-1} t^{-d-\alpha} d t,
$$

where $c_{1}$ and $c_{2}$ are some constants depending on $d$ and $\alpha$. Let $R>0$ satisfy

$$
2 c_{2} \int_{0}^{R}(f(t))^{d+\alpha-1} t^{-d-\alpha} d t<1
$$

so that

$$
M(r) \leq c_{1}+(1 / 2) \sup _{(2 r, R)} M+c_{2} I_{f} \sup _{(R, 1)} M .
$$

It follows that $M$ is bounded by $2 c_{1}+2 c_{2} I_{f} \sup _{(R, 1)} M$. By using the definition of $M$, we conclude that $\Lambda_{0}\left(s_{D_{f}}\right)$ is finite.

We note that by Fatou's lemma, if $y \in \partial_{M} D$ then $P_{D}(x, z) \rightarrow P_{D}(x, y)=\infty$ as $\bar{D}^{c} \ni z \rightarrow y$. If $y \in \partial D \backslash \partial_{M} D$ and $\bar{D}^{c} \ni z \rightarrow y$ non-tangentially (i.e. $|z-y| \leq c \operatorname{dist}(z, D)$ for some $c>0$ ) then by dominated convergence we have $P_{D}(x, z) \rightarrow P_{D}(x, y)<\infty$.

The next result is an extension of [10, Lemma 7].

Proposition 3 If $y \in \partial_{M} D \cap\left((\bar{D})^{c}\right)^{*}$ then

$$
M_{D}(x, y)=\lim _{\bar{D}^{c} \ni z \rightarrow y} \frac{P_{D}(x, z)}{P_{D}\left(x_{0}, z\right)} .
$$

Proof: Suppose that $y=0$ is a limit point of $D$ and of the interior of $D^{c}$, and 0 is accessible for $D$. Let $x \in D$. Recall our notation $D_{r}=D \cap B_{r}, D_{r}^{\prime}=D \backslash D_{r}$. Let $0<4 r<|x| \wedge\left|x_{0}\right|$, and let $z \in B_{r} \backslash \bar{D}$. By Lemma 7 and Remark 2

$$
\int_{D_{r}} G_{D}(x, v) \nu(v, z) d v \geq C_{d, \alpha} \int_{D_{3 r}} s_{D_{2 r}}(v) \nu(v, y) d v \int_{D \backslash D_{2 r}} G_{D}(x, v) \nu(v, y) d v .
$$

This also holds for $x=x_{0}$. By Fatou's lemma we have $\lim _{\bar{D}^{c} \ni z \rightarrow 0} \int_{D_{r}} s_{D_{2 r}}(v) \nu(v, z) d v=$ $\infty$. Since $\int_{D \backslash D_{r}} G_{D}(x, v) \nu(v, z) d v$ is bounded in $z$, (2) yields

$$
\lim _{\bar{D}^{c} \ni z \rightarrow 0} \frac{P_{D}(x, z)}{P_{D}\left(x_{0}, z\right)}=\lim _{\bar{D}^{c} \ni z \rightarrow 0} \frac{\int_{D_{r}} G_{D}(x, v) \nu(v, z) d v}{\int_{D_{r}} G_{D}\left(x_{0}, v\right) \nu(v, z) d v},
$$


provided that limits exist. If $\delta>0$ then for sufficiently small $r$ by (8) we obtain

$$
M_{D}(x, 0)-\delta \leq \frac{\int_{D_{r}} G_{D}(x, v) \nu(v, z) d v}{\int_{D_{r}} G_{D}\left(x_{0}, v\right) \nu(v, z) d v} \leq M_{D}(x, 0)+\delta,
$$

which proves (88). For general $y \in \partial D$ we use translation invariance. If $y=\infty$ then we use inversion. Namely, (2) and (80) and $|T x-T z|=|x-z| /(|x||z|)$ lead to

$$
P_{D}(x, z)=|x|^{\alpha-d}|z|^{-\alpha-d} P_{T D}(T x, T z),
$$

see [17]. This, and (81) yield (88).

If $D=B(0, r), r>0$, and $x_{0}=0$, then we have

$$
M_{D}(x, y)=r^{d-\alpha} \frac{\left(r^{2}-|x|^{2}\right)^{\alpha / 2}}{|x-y|^{d}}, \quad|x|<r,
$$

for every $y \in \partial B(0, r)$. (89) follows from Proposition 3 and (3) or (8) and (23). The formula was given before in [26], [10], [21]. We note that $B_{r}$ has all its boundary points $y$ accessible because $G_{B_{r}}(x, v) \approx(r-|v|)^{\alpha / 2}$ as $B_{r} \ni v \rightarrow y$, see (23). More generally, a Lipschitz (or even $\kappa$-fat) domain has all its boundary points accessible, as follows from [9] ([41]). For more information on the boundary potential theory in Lipschitz domains we refer to the papers [10], [4], [35], [41], which may suggest further applications.

Remark 10 The statement of uniqueness in Theorem 3 may be strengthened. Namely, nonnegative measures $\mu$ on $\partial_{M} D$ and $\lambda$ on $D_{M}^{c}$ are uniquely determined by the values of

$$
\int_{D_{M}^{c}} P_{D}(x, y) \lambda(d y)+\int_{\partial_{M} D} M_{D}(x, y) \mu(d y), \quad \text { for } x \in D,
$$

provided they are finite for (some, hence for all) $x \in D$. This follows from Theorem 4.2 in [21], which states that a (genuine) function $\alpha$-harmonic on open $U \neq \emptyset$ is determined a.e. on $\mathbf{R}^{d}$ by its values on $U$. By a convolution with smooth compactly supported approximate identity (integrability follows from (60)), this yields uniqueness of $\lambda$ and $\mu$ on $\mathbf{R}^{d}$. If $\infty \in \partial_{M} D$, and $\mu$ has an atom at $\infty$ then the mass of the atom is determined by the values of $M_{D}(x, \infty) \mu(\{\infty\})$.

Remark 11 Theorem 4 extends to general (nonnegative) harmonic functions such that measures $\lambda$ and $\mu$ in the representation (9) do not charge $G \cap D^{c}$ in Theorem 4 . To be specific, Theorem 4 applies to $P_{D}[\lambda]+M_{D}[\mu]$ in (9). Indeed, by (77), for finite $y \in \partial_{M} D$ we can consider $M_{D}(\cdot, y)$ a Poisson integral on a wide class of subdomains $U \subset D$ such that $\operatorname{dist}(y, U)>0$, see $(22)$ in this connection. A similar property holds for $M_{D}(\cdot, \infty)$ on on a wide class of bounded domains, if $\infty \in \partial_{M} D$. This follows from the transformation rules for the harmonic measure under the inversion of the domain, see [17].

In domain with regular geometry, e.g. Lipschitz, the condition $(\lambda+\mu)\left(G \cap D^{c}\right)=0$ is equivalent to the traditional assumption of continuity and vanishing of a harmonic function on this set, see [9], [10] and the references there. Continuous decay of harmonic functions cannot, however, be required at irregular points of a domain. 
We finally wish to provide probabilistic interpretations of our results. For a general perspective on probabilistic potential theory and for probabilistic notions mentioned below we refer the reader to, e.g., [23], [7], [14] and [13]. Here we will only indicate a few specific interpretations as they may suggest further extensions.

The second term on the right hand side of (9) is coined singular $\alpha$-harmonic in [10], [21] and [36] (when set to zero on $D^{c}$ ). As explained in [21], in the case of Lipschitz $D$ the function is harmonic for the isotropic $\alpha$-stable Lévy process killed on leaving $D$ (see also [36]). For a general domain $D$ it is more appropriate to relate such functions to the continuous exit of the trajectory of the process from $D$. The observation is implicit in [36]. For example, if $D=B \backslash F$, where $F$ is a non-polar set of Lebesgue measure 0 , then the trajectory of the process is almost surely continuous when entering $F$. Correspondingly, the harmonic measure of $F$ with respect to $D, x \mapsto \omega_{D}^{x}(F)$, is represented with $\lambda=0$ in (9).

The first term in (9) is related to the effect of leaving the domain by a jump. The observation is implicit in [36, formula (5)]. This explains the role of (27) in our development: the second term on the right hand side of (27) is the integral against this part of the harmonic measure, say $\omega_{D-}^{x}$, which results from the jumps of the trajectory from $D$ to $D^{c}$, and the Poisson kernel is the density function of the measure. The latter claim may be verified by using quasi-left continuity of the process $([9])$. The reader may want to consider domains $D$ with $\partial D$ of positive Lebesgue measure, to apprehend the complexity of the relation between $\omega_{D}$ and $\omega_{D-}$. The relation is addressed in Proposition 1 above. In this connection we also refer the reader to [42] for a discussion of the important problem of characterization of domains $D$ for which $\omega_{D}=\omega_{D-}$.

The above complex behavior (jumps and continuous exit) is manifested only for jump processes, which are exemplified here by the isotropic $\alpha$-stable Lévy process on $\mathbf{R}^{d}$. In the presence of jumps the distribution of the position of the process stopped when leaving the domain, i.e. the harmonic measure, is supported on $D^{c}$, but usually not on $\partial D$, and so it is different from the distribution of the position of the process immediately before leaving the domain. The formula of Ikeda and Watanabe in its full form (for which see, e.g., $[28,13,14])$ gives the joint distribution of these two random variables in terms of the Green function and the Lévy measure. Thanks to the simplicity of the Lévy measure $\nu(x, y)$ in $(21)$, the estimates for nonnegative $\alpha$-harmonic functions can be effectively reduced to the estimates of the Green function. We conjecture that (9) generalizes to a wide class of Markov processes of jump type.

For a Riesz type representation of superharmonic functions of the fractional Laplacian on Lipschitz domains we also refer the reader to [21].

We finally wish to provide the following probabilistic connection. The (accessibility) condition $\Lambda_{x}\left(s_{D}\right)=\infty$ has appeared implicitly in [19] and explicitly in [43]. Authors of these papers consider the following property of our symmetric $\alpha$-stable Lévy process $\left\{X_{t}\right\}$ in $\mathbf{R}^{d}$ and a given domain $D$ : There exists a random time interval $\left(\tau_{0}, \tau_{0}+1\right)$ such that $X(t)-X\left(\tau_{0}\right) \in D$ for all $t \in\left(\tau_{0}, \tau_{0}+1\right)$. If $D$ is a thorn then the property holds if and only if $\Lambda_{0}\left(s_{D}\right)=\infty$ ([19]). In [43] all open sets $D$ are considered and the existence of such interval is established if $\Lambda_{0}\left(s_{D}\right)$ is infinite. We conjecture that the accessibility of 0 from $D$ is actually a characterization of this property related to the continuous convergence to 0 of the trajectories of the corresponding conditional process at its lifetime, see [13]. 
Acknowledgements. We thank Krzysztof Samotij for his contribution to our understanding of Martin representation of $\alpha$-harmonic functions. The first named author gratefully acknowledges the hospitality of the Department of Statistics at Purdue University, where the paper was written in part. We thank Krzysztof Burdzy for remarks on accessibility. We thank the referees for many valuable comments and suggestions.

\section{References}

[1] H. Aikawa, Boundary Harnack principle and Martin boundary for a uniform domain. J. Math. Soc. Japan 53 (2001), no. 1, 119-145.

[2] H. Aikawa, Potential-theoretic characterizations of nonsmooth domains. Bull. London Math. Soc. 36 (2004), no. 4, 469-482.

[3] D. Armitage, S. Gardiner, Classical potential theory. Springer-Verlag, London, 2001.

[4] R. Bañuelos, K. Bogdan, Symmetric stable processes in cones. Potential Anal. 21 (2004), no. 3, 263-288.

[5] R. Bass, Probabilistic techniques in analysis Springer-Verlag, New York, 1995.

[6] R. Bass, K. Burdzy, A probabilistic proof of the boundary Harnack principle. Seminar on Stochastic Processes, 1989 (San Diego, CA, 1989) 1-16, Progr. Probab., 18, Birkhuser Boston, Boston, MA, 1990.

[7] J. Bliedtner, W. Hansen, Potential Theory. An analytic and probabilistic approach to balayage Springer-Verlag Berlin Heidelberg, 1986.

[8] R. M. Blumenthal, R. K. Getoor, D. B. Ray. On the distribution of first hits for the symmetric stable processes. Trans. Amer. Math. Soc. 99 (1961), 540-554.

[9] K. Bogdan, The boundary Harnack principle for the fractional Laplacian. Studia Math. 123 (1997), 43-80.

[10] K. Bogdan, Representation of $\alpha$-harmonic functions in Lipschitz domains. Hiroshima Math. J. 29 (1999), 227-243.

[11] K. Bogdan, Sharp estimates for the Green function in Lipschitz domains. J. Math. Anal. Appl. 243 (2000), no. 2, 326-337.

[12] K. Bogdan, K. Burdzy, Z.-Q. Chen, Censored stable processes. Probab. Theory Related Fields 127 (2003), no. 1, 89-152.

[13] K. Bogdan and T. Byczkowski, Potential theory for the $\alpha$-stable Schrödinger operator on bounded Lipschitz domains. Studia Math., 133 (1999), no. 1, 53-92.

[14] K. Bogdan, T. Byczkowski, Potential theory of Schrödinger operator based on fractional Laplacian. Probab. Math. Statist. 20 (2000), no. 2, Acta Univ. Wratislav. No. 2256, 293-335. 
[15] K. Bogdan, A. Stós, P. Sztonyk, Harnack inequality for stable processes on d-sets. Studia Math. 158 (2003), no. 2, 163-198.

[16] K. Bogdan, P. Sztonyk, Estimates of potential kernel and Harnack's inequality for anisotropic fractional Laplacian. Preprint (2005) http://arxiv.org/abs/math.PR/0507579

[17] K. Bogdan, T. Żak, On Kelvin transformation. J. Theor. Prob. 19 (2006), no. 1, $89-120$.

[18] M. Brelot, On topologies and boundaries in potential theory, Lecture Notes in Mathematics, Springer, Berlin, 1971.

[19] K. Burdzy, T. Kulczycki, Stable processes have thorns. Ann. Probab. 31 (2003), 170-194

[20] Z.-Q. Chen, P. Kim, Green function estimate for censored stable processes. Probab. Theory Related Fields 124 (2002), no. 4, 595-610.

[21] Z.-Q. Chen, R. Song, Martin boundary and integral representation for harmonic functions of symmetric stable processes. J. Funct. Anal. 159 (1998), 267-294.

[22] Z.-Q. Chen, R. Song, Conditional gauge theorem for non-local Feynman-Kac transforms. Probab. Theory Relat. Fields 125 (2003), 45-72.

[23] K. Chung, Z. Zhao, From Brownian motion to Schrödinger's equation. SpringerVerlag, New York, 1995.

[24] W. Hansen, Uniform boundary Harnack principle and generalized triangle property. J. Funct. Anal. 226 (2005), no. 2, 452-484.

[25] W. Hansen, Global comparison of perturbed Green functions. Math. Ann. 334 (2006), no. 3, 643-678.

[26] F. Hmissi, Fonctions harmoniques pour les potentiels de Riesz sur la boule unit (French) [Harmonic functions for Riesz potentials on the unit ball]. Exposition. Math. 12 (1994), no. 3, 281-288.

[27] R. A. Hunt, R. L. Wheeden, Positive harmonic functions on Lipschitz domains. Trans. Amer. Math. Soc. 147 (1970), 507-527.

[28] N. Ikeda, S. Watanabe, On some relations between the harmonic measure and the Lévy measure for a certain class of Markov processes. Probab. Theory Related Fields 114 (1962), 207-227.

[29] N. Jacob, Pseudo differential operators and Markov processes. Vol. I, II, III. Imperial College Press, London, 2001-2005.

[30] T. Jakubowski, The estimates for the Green function in Lipschitz domains for the symmetric stable processes. Probab. Math. Statist. 22 (2002), no. 2, Acta Univ. Wratislav. No. 2470, 419-441. 
[31] T. Kulczycki, Properties of Green function of symmetric stable processes. Probab. Math. Statist. 17 (1997), no. 2, Acta Univ. Wratislav. No. 2029, 339-364.

[32] T. Kulczycki, Intrinsic ultracontractivity for symmetric stable processes. Bull. Polish Acad. Sci. Math. 46 (1998), no. 3, 325-334.

[33] H. Kunita, T. Watanabe, Markov processes and Martin boundaries I. Illinois J. Math. 9 (1965), 485-526.

[34] N. S. Landkof, Foundations of modern potential theory. Springer-Verlag, New YorkHeidelberg 1972.

[35] K. Michalik, M. Ryznar, Relative Fatou theorem for $\alpha$-harmonic functions in Lipschitz domains. Illinois J. Math. 48 (2004), no. 3, 977-998.

[36] K. Michalik, K. Samotij, Martin representation for $\alpha$-harmonic functions. Probab. Math. Statist. 20 (2000), 75-91.

[37] R. Pinsky, Positive harmonic functions and diffusion Cambridge Studies in Advanced Mathematics, 45. Cambridge University Press, Cambridge, 1995.

[38] S. Port, Hitting times and potentials for recurrent stable processes. J. Analyse Math. 20 (1967), 371-395.

[39] M. Riesz, Intégrales de Riemann-Liouville et potentiels. Acta Sci. Math. Szeged, 1938.

[40] K. Sato, Lévy processes and infinitely divisible distributions. Cambridge Univ. Press, Cambridge, 1999.

[41] R. Song, J.-M. Wu, Boundary Harnack principle for symmetric stable processes. J. Funct. Anal. 168 (1999), 403-427

[42] J.-M. Wu, Harmonic measures for symmetric stable processes. Studia Math. 149 (2002), no. 3, 281-293.

[43] J.-M. Wu, Symmetric stable processes stay in thick sets. Ann. Probab. 32 (2004), $315-336$.

Krzysztof Bogdan (bogdan@pwr.wroc.pl)

Department of Statistics, Purdue University and

Institute of Mathematics and Computer Science,

Wrocław University of Technology

ul. Wybrzeże Wyspiańskiego 27, 50-370, Wrocław, Poland

Tadeusz Kulczycki (Tadeusz.Kulczycki@pwr.wroc.pl),

Mateusz Kwaśnicki (Mateusz.Kwasnicki@pwr.wroc.pl)

Institute of Mathematics and Computer Science

Wrocław University of Technology

ul. Wybrzeże Wyspiańskiego 27, 50-370, Wrocław, Poland 